\title{
String theory origin of bipartite SCFTs
}

\section{Citation}

Heckman, Jonathan J., Cumrun Vafa, Dan Xie, and Masahito Yamazaki. 2013. “String Theory Origin of Bipartite SCFTs." Journal of High Energy Physics 2013 (5). https://doi.org/10.1007/ jhep05(2013)148.

\section{Permanent link}

http://nrs.harvard.edu/urn-3:HUL.InstRepos:41385028

\section{Terms of Use}

This article was downloaded from Harvard University's DASH repository, and is made available under the terms and conditions applicable to Open Access Policy Articles, as set forth at http:// nrs.harvard.edu/urn-3:HUL.InstRepos:dash.current.terms-of-use\#OAP

\section{Share Your Story}

The Harvard community has made this article openly available.

Please share how this access benefits you. Submit a story.

\section{Accessibility}




\title{
String Theory Origin of Bipartite SCFTs
}

\author{
Jonathan J. Heckman ${ }^{1 *}$, Cumrun Vafa ${ }^{1 \dagger}$, \\ Dan $\mathrm{Xie}^{2 \ddagger}$ and Masahito Yamazaki ${ }^{3 \S}$ \\ ${ }^{1}$ Jefferson Physical Laboratory, Harvard University, Cambridge, MA 02138, USA \\ ${ }^{2}$ School of Natural Sciences, Institute for Advanced Study, Princeton, NJ 08540, USA \\ ${ }^{3}$ Department of Physics, Princeton University, Princeton, NJ 08544, USA
}

\begin{abstract}
We provide a string theory embedding for $\mathcal{N}=1$ superconformal field theories defined by bipartite graphs inscribed on a disk. We realize these theories by exploiting the close connection with related $\mathcal{N}=2$ generalized $\left(A_{k-1}, A_{n-1}\right)$ Argyres-Douglas theories. The $\mathcal{N}=1$ theory is obtained from spacetime filling D5-branes wrapped on an algebraic curve and NS5-branes wrapped on special Lagrangians of $\mathbb{C}^{2}$ which intersect along the BPS flow lines of the corresponding $\mathcal{N}=2$ Argyres-Douglas theory. Dualities of the $\mathcal{N}=1$ field theory follow from geometric deformations of the brane configuration which leave the UV boundary conditions fixed. In particular we show how to recover the classification of IR fixed points from cells of the totally non-negative Grassmannian $\mathrm{Gr}_{k, n+k}^{\mathrm{tnn}}$. Additionally, we present evidence that in the 3D theory obtained from dimensional reduction on a circle, VEVs of line operators given by D3-branes wrapped over faces of the bipartite graph specify a coordinate system for $\operatorname{Gr}_{k, n+k}^{\mathrm{tnn}}$.
\end{abstract}

November 2012

\footnotetext{
*e-mail: jheckman@physics.harvard.edu

$\dagger$ e-mail: vafa@physics.harvard.edu

$\ddagger$ e-mail: dxie@ias.edu

$\S$ e-mail: masahito@princeton.edu
} 


\section{Contents}

1 Introduction $\quad 2$

2 Bipartite $\mathcal{N}=1$ SCFTs

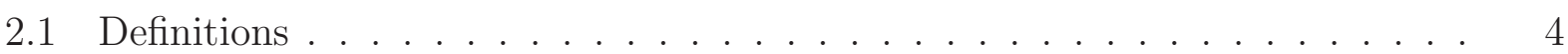

2.2 IR Equivalence Classes and the Grassmannian . . . . . . . . . . . . . . 5

3 The Top Cell of $\mathrm{Gr}_{k, n+k}^{\mathrm{tnn}}$

3.1 The Quivers .......................... 8

3.2 Infrared R-Symmetry . . . . . . . . . . . . . . . . . . 9

4 Bipartite Graphs and $\mathcal{N}=2$ BPS Quivers 14

5 Stringy Realization $\quad 17$

5.1 The $\mathrm{Gr}_{2, n+2}^{\mathrm{tnn}}$ Theories . . . . . . . . . . . . . . . . . 20 20

5.2 The $\mathrm{Gr}_{k, n+k}^{\mathrm{tnn}}$ Theories . . . . . . . . . . . . . . . . 24 24

5.3 UV Boundary Conditions . . . . . . . . . . . . . . . . 27

5.4 Reproducing the Square Move . . . . . . . . . . . . . . . . 28

6 Physical Origin of Coordinates for $\mathrm{Gr}_{k, n+k}^{\mathrm{tnn}}$

7 Conclusions

A Coordinates of $\mathrm{Gr}_{k, n+k}^{\mathrm{tnn}}$

B Construction of Special Lagrangians

B.1 Existence and Uniqueness . . . . . . . . . . . . . . . . . 37

B.2 Explicit Example . . . . . . . . . . . . . . . . . . . . 39 


\section{Introduction}

A striking feature of string theory is the simple geometric characterization it provides of diverse quantum field theories in various dimensions. Non-trivial field theoretic dualities often have a simple geometric underpinning in the string realization. Indeed, the rich mathematical structure of string geometry often reveals itself in surprisingly diverse ways in an effective field theory.

Recently a new class of $\mathcal{N}=1$ quantum field theories was discovered which are specified by planar bipartite graphs inscribed on a disk [1] This class of theories is related to, but distinct from, earlier work on quiver gauge theories specified by a dimer on a torus [2 5] (see [6],7] for reviews). Given such a bipartite graph, there is a corresponding quiver gauge theory with gauge groups, matter fields and superpotential interaction terms specified by the connectivity of the graph. While these theories are non-conformal in the UV, it was conjectured in [1] that they flow to non-trivial strongly coupled IR fixed points. Moreover, thanks to the mathematical results of [8] for bicolored graphs, these fixed points are classified by decorated permutations, which in turn are specified by cells in the totally non-negative Grassmannian $\mathrm{Gr}_{k, n+k}^{\mathrm{tnn}}$. Here the totally non-negative part (tnn) of the Grassmannian denotes the subspace of $\operatorname{Gr}_{k, n+k} \equiv G L_{k}(\mathbb{R}) \backslash \operatorname{Mat}_{k, n+k}(\mathbb{R})$ with all Plücker coordinates non-negative. Closely related but distinct bipartite theories have been considered in [9].

The specification of a cell in the totally non-negative Grassmannian provides a simple way to characterize possible Seiberg dual phases, that is, the equivalence class of bipartite networks which define the same IR fixed point. This can be checked by appealing to the available results in the mathematics literature, but it leaves open the underlying question as to why these equivalences should hold, and more generally, the physical origin of these bipartite graphs.

In this paper we provide an embedding in string theory for such bipartite theories. Additionally, we show how lower order deformations of the brane configuration translate to non-trivial field theoretic dualities, thus providing a geometric characterization of Seiberg dual theories. Moreover, we give a map between the coordinates of the Grassmannian and operators of these $\mathcal{N}=1$ theories.

Our $\mathcal{N}=1$ theories are realized by a configuration of spacetime filling D5-branes and NS5-branes in type IIB string theory. The background geometry is given by $\mathbb{R}^{3,1} \times \mathbb{C}^{2} \times \mathbb{C}_{\perp}$, with all branes sitting at the same point of $\mathbb{C}_{\perp}$. In this geometry, we consider a stack of $N$

\footnotetext{
${ }^{1} \mathrm{~A}$ bicolored network or graph is a graph in which each vertex can be colored as black or white. A bipartite graph or "dimer" refers to the case where each vertex only attaches to a vertex of the opposite coloring. In the physical theory the bipartite and bicolored graphs define the same physical theory so we shall often use the two terms interchangeably.
} 
D5-branes wrapping the algebraic curve $\Sigma$ defined as the zero set in $\mathbb{C}^{2}$ of:

$$
y^{k}=x^{n}+\text { (deformations) },
$$

together with NS5-branes wrapping special Lagrangian (sLag) submanifolds of $\mathbb{C}^{2}$ which intersect $\Sigma$ along one-dimensional subspaces. This intersection pattern traces out a network of one-cycles on $\Sigma$ which partitions the D5-brane into separate pieces, realizing the quiver gauge theories for the top-dimensional cell of $\mathrm{Gr}_{k, n+k}^{\mathrm{tnn}}$. We reach the lower cells by brane recombination, corresponding to a partial Higgsing of the quiver gauge theory.

Although this construction yields an $\mathcal{N}=1$ supersymmetric theory, the geometric ingredients follow from an $\mathcal{N}=2$ precursor 2 For example, the curve $\Sigma$ can also be wrapped by an M5-brane, realizing a 4D $\mathcal{N}=2$ theory. In the limit where all deformations are switched off, this produces the $\mathcal{N}=2$ generalized $\left(A_{k-1}, A_{n-1}\right)$ Argyres-Douglas fixed point (see for example Figure 10 for the comparison between $4 \mathrm{~d} \mathcal{N}=1$ theory and $\mathcal{N}=2$ theory). Once the deformations of the geometry are switched on, the one-cycles of the intersection correspond to a network of BPS flows which preserve a fixed $\mathcal{N}=1$ subalgebra of the $\mathcal{N}=2$ supersymmetry algebra. Given such a one-dimensional network, there exists a unique set of special Lagrangians in $\mathbb{C}^{2}$ which intersect $\Sigma$, realizing our quiver gauge theories.

The geometric characterization of these quiver gauge theories also allows us to delineate possible Seiberg dual phases. Fixing the boundary conditions of all of the branes, lower order deformations of the algebraic curve $\Sigma$ translate to possible jumps in the connectivity of the BPS flow lines. In the $\mathcal{N}=1$ quiver gauge theory this corresponds to Seiberg duality.

The string construction also leads us to a conjectural relation between the coordinates of $\mathrm{Gr}_{k, n+k}^{\mathrm{tnn}}$ and the VEVs of line operators in the dimensional reduction of the $4 \mathrm{D}$ theory. The basic correspondence involves D3-branes suspended between NS5-branes which are wrapped over two directions of the $4 \mathrm{D}$ spacetime. Compactifying the $4 \mathrm{D}$ spacetime on the geometry $S_{(t)}^{1} \times M C_{q}$ with $M C_{q}$ a Melvin cigar geometry, we show that in the small $S_{(t)}^{1}$ limit, these operators are closely related to line operators of the $\mathcal{N}=2$ Argyres-Douglas theory. Quite remarkably, this class of line operators specify coordinates of the Grassmannian.

The rest of this paper is organized as follows. In section 2 we review the defining elements of the bipartite quiver gauge theories and the correspondence with the totally non-negative Grassmannian. In section 3 we discuss in more detail the theories defined by the top cell of $\mathrm{Gr}_{k, n+k}^{\mathrm{tnn}}$, since all other fixed points follow from Higgsing of this case. In preparation for our discussion of $\mathcal{N}=1$ SCFTs, in section 4 we review some aspects of BPS quivers for $\mathcal{N}=2$ theories, and in particular the close connection between the bipartite graphs for $\mathrm{Gr}_{k, n+k}^{\mathrm{tnn}}$ and the generalized $\left(A_{k-1}, A_{n-1}\right)$ Argyres-Douglas theories. In section 5 we present our proposal

\footnotetext{
${ }^{2}$ A similar correspondence has been studied for bipartite graphs on a torus [10, where the same geometry and the same quiver describe either a four-dimensional $\mathcal{N}=1 \mathrm{SCFT}$, or a one-dimensional quantum mechanics for a $1 / 2$ BPS particle inside a four-dimensional $\mathcal{N}=2$ theory.
} 
for a string theory realization of these quiver gauge theories. Moreover, we show that this construction automatically explains the fact that various gauge theories defined by related bipartite networks flow to the same IR fixed point. In section 6 we explain how in the compactification down to three dimensions, line operators of the theory specify coordinates of the Grassmannian. Section 7 presents our conclusions and possible directions of future investigation. Some additional technical details are deferred to the Appendices.

\section{Bipartite $\mathcal{N}=1$ SCFTs}

In this section we introduce a class of quiver gauge theories defined by planar bipartite networks [1]. We first review the basic properties of these theories and then explain how the combinatorics of the graphs can be related to a cell decomposition of the totally non-negative Grassmannian. This provides a powerful way to characterize possible IR fixed points of the quiver gauge theories. One of our tasks in this paper will be to give a simple explanation for these relations from a brane construction in string theory.

\subsection{Definitions}

To frame the discussion to follow we begin by defining in more precise terms our bipartite theories. A bicolored network (or "graph") is a graph in which the vertices can be colored as either black or white. A bipartite graph or dimer refers to a further specialization where only black vertices are adjacent to white vertices and visa versa. Throughout this paper we restrict attention to graphs which are connected and can be drawn in the plane, and in which all legs which attach to only one vertex are external, that is, they can be drawn as ending on a circle at infinity which surrounds the planar graph.

Given such a bicolored graph we associate a $4 \mathrm{D} \mathcal{N}=1$ quiver gauge theory to it by taking the graph dual. In more detail:

1. Attach a $U(N)$ gauge group to each closed, i.e. compact face and a $U(N)$ flavor group to an open, i.e. non-compact face on the boundary 3 These are represented in figure 1 by red circles and squares, respectively.

2. Each edge gives a bifundamental field. The chirality of the bifundamental, or equivalently the orientation of the arrow of the quiver diagram, is determined by the black/white coloring of the vertices; the quiver arrows form a clockwise (resp. counterclockwise) loop around the black (resp. white) vertices. There is an important exception to this rule: we do not associate a bifundamental matter to external edges which attach to a white vertex.

\footnotetext{
${ }^{3}$ We could consider more general theories by choosing different ranks for different faces, as long as they are anomaly free.
} 

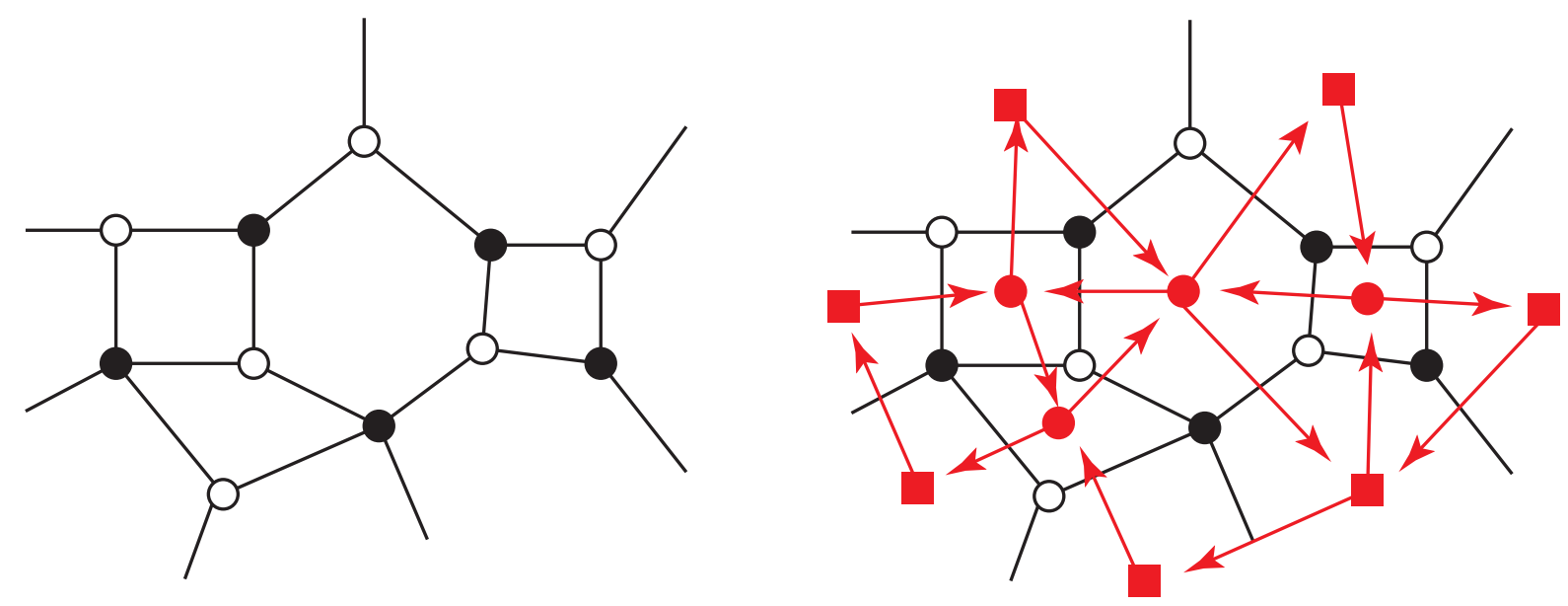

Figure 1: Depiction of a planar bicolored graph, corresponding to a cell of $\mathrm{Gr}_{3,7}^{\mathrm{tnn}}$. The dual graph defines a $4 \mathrm{D} \mathcal{N}=1$ quiver gauge theory. In the original bicolored graph, each face specifies either a gauge group (red circle) or a flavor group (red square). Each interior edge specifies an oriented bifundamental, and each interior vertex defines a superpotential term. For exterior edges and vertices there is only a contribution from black vertices.

3. There is a superpotential term for each vertex, given by the trace of the product of all the bifundamentals around the vertex.

Note that in this construction, all of the gauge symmetries, as well as flavor symmetries, are automatically anomaly free. This is because there are an equal number of ingoing and outgoing arrows.

The rules above are chosen such that the mathematical result of [8] can be directly translated into physical statements about the IR fixed points of the $4 \mathrm{D} \mathcal{N}=1$ theories. The rules mostly follow the string theory literature on dimers on a torus [2, 3]. However as we already mentioned, there is one crucial difference due to the fact that white vertices attached to an external leg are treated differently. Note that the paper of [9] does not include this rule, and hence our theory is different from the theory defined there. Our definition is chosen so that the square move of the bipartite graph corresponds to Seiberg duality of the $4 \mathrm{D} \mathcal{N}=1$ theory, even for faces which are adjacent to flavor branes.

One of our aims in this paper will be to explain how these seemingly ad hoc conditions naturally arise in a string construction (see in particular the discussion around Figure 11).

\subsection{IR Equivalence Classes and the Grassmannian}

Having introduced our quiver gauge theories, it is natural to study their properties in the infrared. In the remainder of this section we explain how the geometry of the Grassmannian naturally arises in a classification of IR fixed points. 
(a)

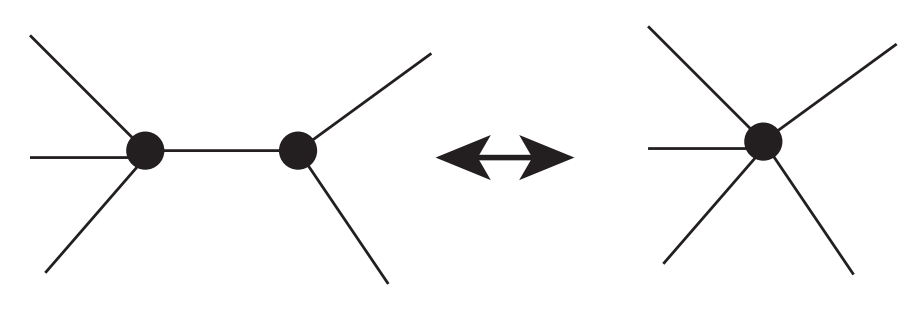

(b)

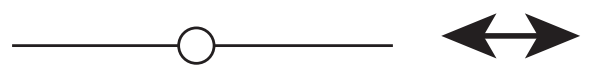

(c)

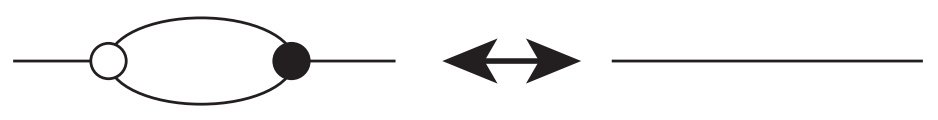

(d)

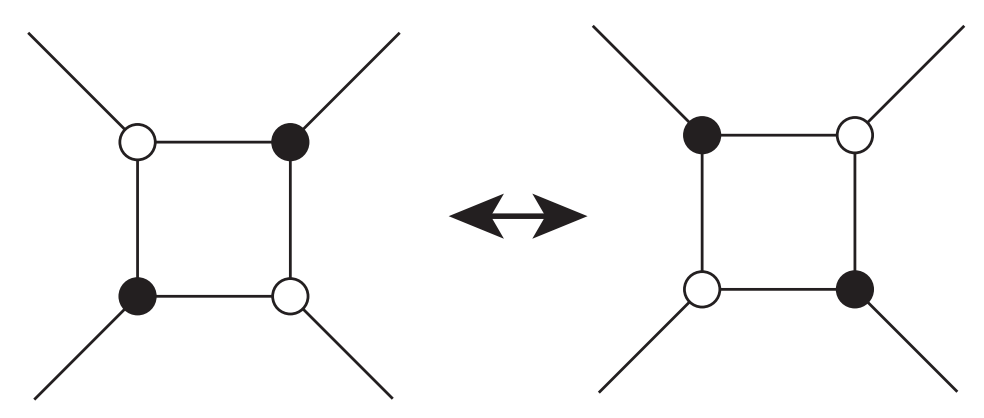

Figure 2: Depiction of graphical operations on a planar bicolored network which preserve the IR fixed point of the associated $4 \mathrm{D} \mathcal{N}=1$ theory. (a,b): integrating out massive matter fields, (c): confinement of a gauge group, (d): Seiberg duality.

Given an $\mathcal{N}=1$ theory defined from a bicolored network, we want to determine the graphical operations which would not change the IR physics. First of all, one could integrate out massive fields in the theory, and such operations are mapped to merging same-colored vertices connected by edges (figure 2(a)) or removing the degree two vertices (figure 2(b)). We can also integrate out the IR-confining gauge group with $N_{f}=N_{c}$, and this operation is mapped graphically to the so-called bubble reduction (figure 2(c)).

Far more non-trivial is the Seiberg duality acting on a gauge group in the conformal window. In our case, this only happens for square faces corresponding to gauge groups with $N_{f}=2 N$. Seiberg duality then corresponds to the so-called "square move" (figure $2(\mathrm{~d})$ ).

It is very useful to restrict to bicolored networks which do not have bubbles under any of the moves introduced earlier. Such a "reduced" graph contains the minimal UV data necessary to characterize the IR fixed point. There is a powerful combinatorial algorithm, based on "zig-zag paths", for determining whether or not a network is reduced [1]. In the following we will restrict to the case where such a reduction has already been performed.

Given two reduced graphs, we can now ask whether these two theories will flow to the 

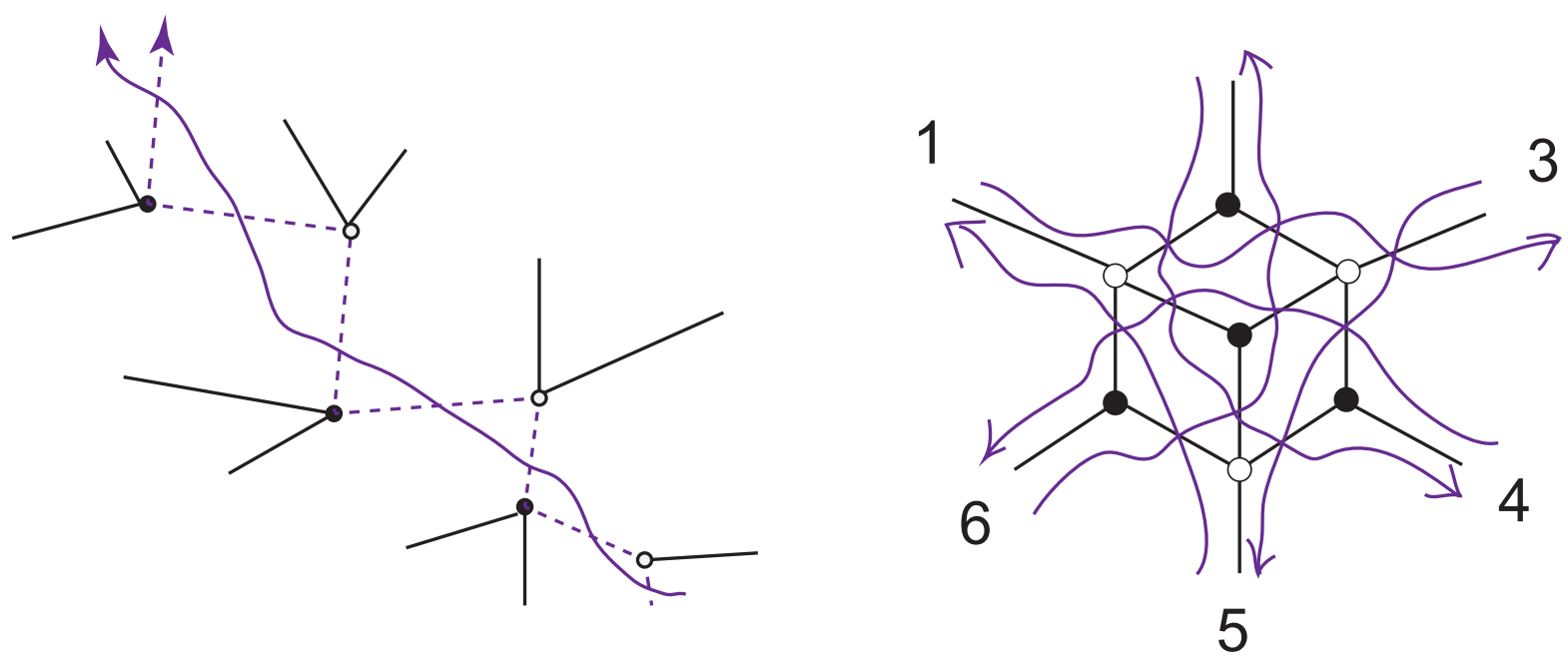

Figure 3: An example of a zig-zag path on a bipartite graph. The rule is specified by following a given leg, and turning left at a white vertex and right at a black vertex (left). For a planar bipartite graph, this yields a permutation assignment between one external leg and another (right).

same IR fixed point. This can also be answered with the help of zig-zag paths, and permutations defined from them. Namely two reduced networks define the same IR SCFT if they define the same permutation.

To define a zig-zag path (or an associated "strand") and a permutation, let us label all of the external legs according to a counter-clockwise cyclic ordering on the boundary circle "at infinity". Starting from a given external leg (say the $i$-th leg) at infinity and following it into the interior region of the graph, we obtain a path on the graph by turning left at the white vertices and right at the black vertices, finally ending at another external leg, say the $j$-th leg (figure 3). Each zig-zag path around the graph defines a permutation $i \rightarrow \pi(i):=j$ of $\mathfrak{S}_{n+k}$, where we have denoted the number of external legs by $n+k$, to match with the notation later.

There is a remarkable correspondence between this permutation and the cell decomposition of a totally non-negative Grassmannian $\mathrm{Gr}_{k, n+k}^{\mathrm{tnn}}$ [8] 4. This means that the IR fixed points of our $\mathcal{N}=1$ theories can also be uniquely labeled by the cells of the $\mathrm{Gr}_{k, n+k}^{\mathrm{tnn}}$. Here the values of $k$ and $n$ can be read off from the formula

$$
k-n=\sum_{\text {black }}(\operatorname{deg}(v)-2)-\sum_{\text {white }}(\operatorname{deg}(v)-2),
$$

\footnotetext{
${ }^{4}$ Each cell is a ball, and is roughly a subspace of the real Grassmannian with some of the Plücker coordinates positive, with the rest set to zero.
} 
where $\operatorname{deg}(v)$ denotes the number of edges attached to a vertex 5

The details of this correspondence are explained in Appendix A. In particular we explain how coordinates of the Grassmannian are mapped to the weights of faces in the bicolored network. In section 6 we give a physical interpretation for these parameters.

\section{The Top Cell of $\mathbf{G r}_{k, n+k}^{\mathrm{tnn}}$}

In this section we discuss in more detail the theories defined by the top-dimensional cell of the totally non-negative Grassmannian $\mathrm{Gr}_{k, n+k}^{\mathrm{tnn}}$. These theories are canonical in the sense that a theory associated with a lower cell (corresponding to going to the boundary of the top cell) can be obtained from partial Higgsing of the top cell theory.

\subsection{The Quivers}

The top-dimensional cell of $\mathrm{Gr}_{k, n+k}^{\mathrm{tnn}}$ is a cell with all the Plücker coordinates positive. This corresponds to a permutation

$$
\pi_{\text {top }}(i)=i+k \quad(\bmod n+k), \quad i=1, \ldots, n+k,
$$

and bicolored graph and corresponding quiver shown in figure 4. We can construct these graphs from (3.1) by use of the algorithm explained in [1,8]. One can check explicitly that the permutation defined from the zig-zag paths is indeed given by (3.1). The face of the network is either a square or hexagon. The total number of $U(N)$ gauge groups is $(n-1)(k-1)$, and the number of flavor groups is $n+k$.

Let us note that the geometric operation which interchanges $k$ and $n$ in the Grassmannian corresponds to switching all black and white vertices. This leaves the connectivity of the internal parts of the quiver unchanged, though it will alter the number of gauge singlets, as we treat external legs attached to black and white vertices differently.

Finally, in the special case where $k=2$, we have a single row of squares with alternating black and white vertices. Each white vertex supports an external leg, but only the leftmost and rightmost black vertices support an external leg. According to our rules specified in section 2, this means there are two gauge singlets. We obtain a distinct quiver gauge theory by interchanging $k$ and $n$. In the graph, this corresponds to switching all black and white vertices, so that there are $n$ gauge singlets for the top cell of $\mathrm{Gr}_{n, n+2}^{\mathrm{tnn}}$.

\footnotetext{
${ }^{5}$ Let us note that although there is a geometric duality which interchanges $k \leftrightarrow n$, these graphs define different IR fixed points.
} 

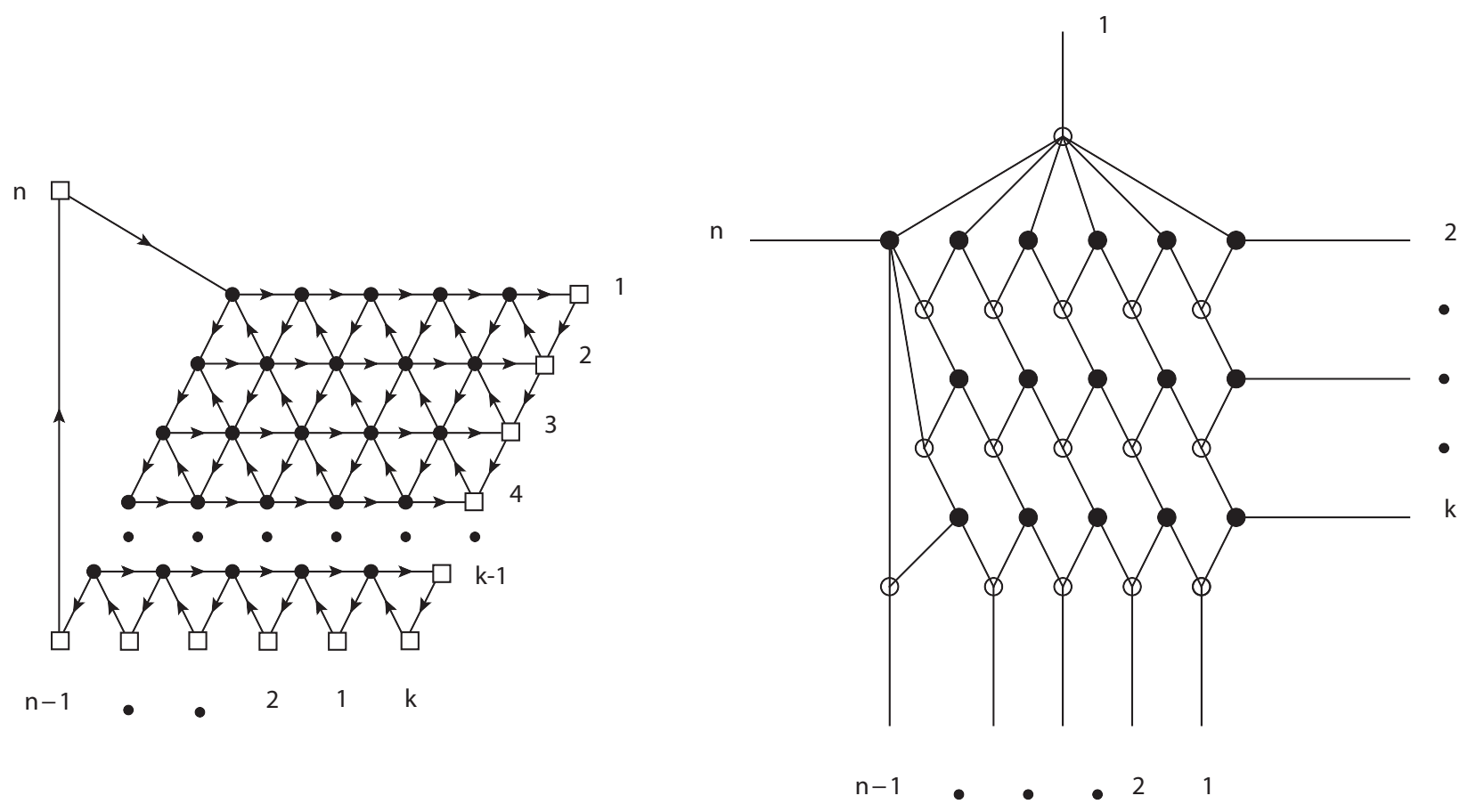

Figure 4: The quiver (left) and the bipartite graph (right) associated with the topdimensional cell of $\mathrm{Gr}_{k, n+k}^{\mathrm{tnn}}$. Square nodes of the quiver diagram denote flavor group factors and correspond to non-compact faces of the bipartite graph.

\section{$3.2 \quad$ Infrared R-Symmetry}

In this subsection we discuss some properties of the infrared fixed points for the theories associated with the top cell of $\mathrm{Gr}_{k, n+k}^{\mathrm{tnn}}$. We begin with some general considerations which apply to all of our bipartite theories. We then present evidence for a surprisingly rich phase structure as a function of $k$ and $n$.

In supersymmetric theories, a wealth of information is encoded in the infrared R-symmetry. This is given by a linear combination of the UV R-symmetry and all abelian flavor symmetries of the IR theory:

$$
R_{I R}=R_{U V}+\sum_{i} t_{i} F_{i} .
$$

The list of such abelian flavor symmetries includes all of the central $U(1) \subset U(N)$ factors of the UV flavor symmetries, but can also include emergent $U(1)$ symmetries. In general, it can be difficult to determine when such accidental $U(1)$ 's appear. One clear indication of their presence is signalled by the existence of an otherwise consistent looking fixed point which nevertheless naively contains an operator with dimension below the unitarity bound. Assuming one has taken into account all such $U(1)$ 's, the procedure of $a$-maximization amounts 
to maximizing a trial $a$-function [1]:

$$
a_{\text {trial }}[t]=\frac{3}{32}\left(3 \operatorname{Tr} R_{I R}^{3}-\operatorname{Tr} R_{I R}\right)
$$

over the $t_{i}$, subject to all constraints imposed by a consistent IR fixed point.

Consider now the procedure of $a$-maximization for our bipartite theories. The analysis is quite similar to the case of torus dimers discussed in [3, 4]. Since we have a weakly coupled UV definition of the theory, we can appeal to anomaly matching considerations to work directly in terms of the UV basis of fields. In a general bipartite theory the constraints on the coefficients $t_{i}$ are specified by two types of conditions. Letting $R(X)$ denote the $\mathrm{R}$-charge of a bifundamental $X$, the vanishing of the NSVZ beta function for each face implies (see e.g. [3, 4]):

$$
\text { For each face } F: \sum_{X \text { on } F}(1-R(X))=2,
$$

where the sum over " $X$ on $F$ " includes all bifundamentals attached to the face $F$. Even in the absence of the superpotential interaction terms, this will often produce a non-trivial fixed point. Adding the superpotential deformations can be viewed as a further deformation of the theory. In order for this deformation to be a relevant/marginal deformation, it must have $\mathrm{R}$-charge +2 in the IR:

$$
\text { For each vertex } V: \sum_{X \text { on } V} R(X)=2 \text {. }
$$

The procedure of $a$-maximization then corresponds to varying with respect to these trial R-charges, subject to the constraints of equations (3.4) and (3.5). We note that in contrast to the case of dimer models on a torus, there can be gauge singlets which participate in only a single superpotential term. When such a gauge singlet multiplies a meson, this meson will vanish in the chiral ring. In other words, such a meson does not parameterize the vacuum structure of the low energy theory.

Let us consider in more detail the analysis of $\mathrm{R}$-charge assignments for the $k=2$ theories, i.e., for the top cell of $\mathrm{Gr}_{2, n+2}^{\mathrm{tnn}}$. Now, as we have already mentioned, one of the key points of the classification by cells of the Grassmannian is that different bipartite graphs may nevertheless flow to the same IR fixed point. Since the flavor symmetries remain the same after Seiberg duality, it is enough to analyze the R-charge assignments in any given chamber. A simple choice of duality frame is the one depicted in figure 5 which consists of a single row of squares (i.e. "diamonds") which share a common vertex.

The flavor symmetries of our system include all of the central $U(1)$ factors of the noncompact flavor faces. This provides $n+2$ flavor symmetries. Additionally, there are $n-1$ "baryonic" flavor symmetries corresponding to the central $U(1)$ attached to each gauge group 

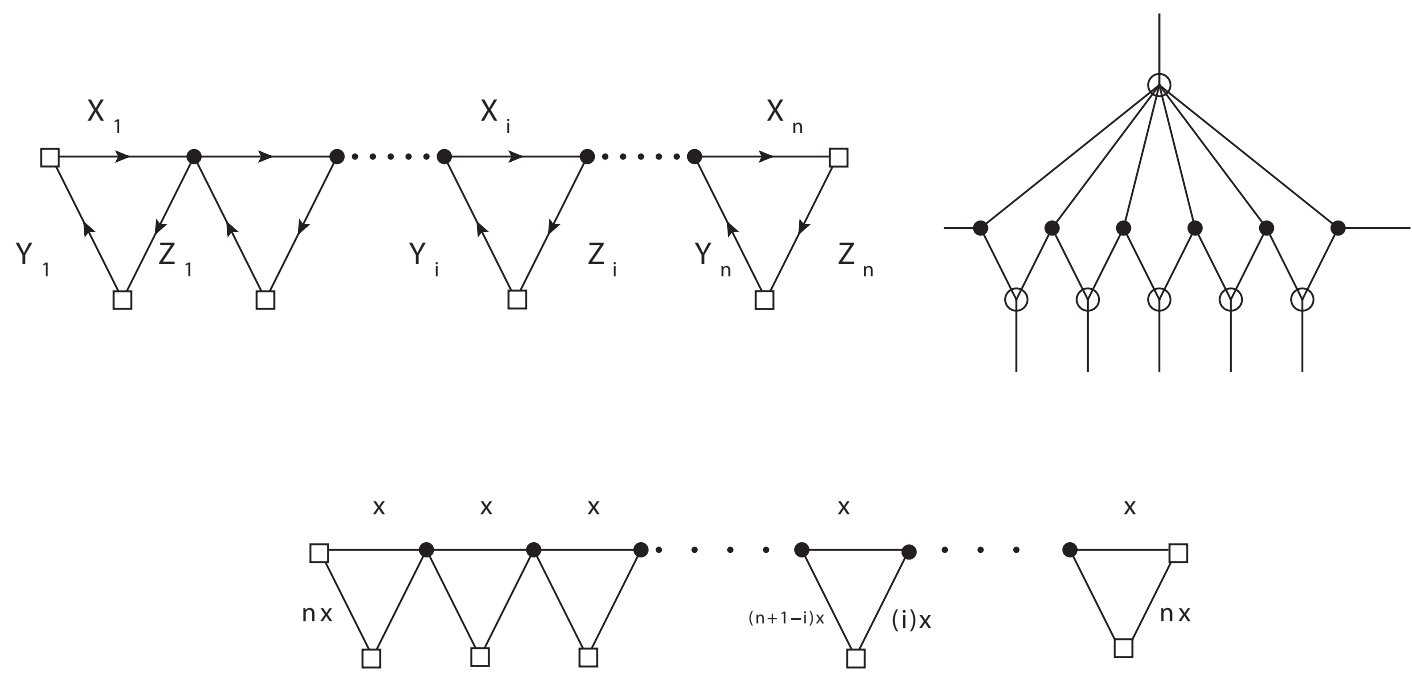

$$
x=2 /(n+2)
$$

Figure 5: The quiver (left), the bipartite graph (right) and the "naive" R-charge assignments (bottom) for the top-dimensional cell of $\mathrm{Gr}_{2, n+2}^{\mathrm{tnn}}$. Square nodes denote flavor group factors. All of the R-charge assignments of bifundamentals are proportional to $x=2 /(n+2)$.

factor. In a theory with gauge group $S U(N)$, these can play the role of flavor symmetries for dibaryon operators of the form:

$$
\mathcal{B}_{G_{i} G_{j}}=\varepsilon \overline{j_{1}}, \ldots, \overline{j_{N}} \varepsilon^{i_{1} \ldots i_{N}} Q_{i_{1}}^{\overline{j_{1}}} \ldots Q_{i_{N}}^{\overline{j_{N}}}
$$

constructed from a bifundamental $Q$ between groups $G_{i}$ and $G_{j}$. This can also happen in our case, because the central $U(1)$ of a gauge group factor decouples in the infrared.

In addition to these UV symmetries, there can also be accidental $U(1)$ symmetries in the infrared theory. We will present some evidence that a self-consistent treatment of these fixed points involves the inclusion of such accidental $U(1)$ 's. To argue for their existence, we first proceed as if they did not exist.

In the duality frame of figure 5, it is convenient to label the bifundamentals according to small triangles formed in the quiver. For $i=1, \ldots, n$, we denote by $X_{i}$ the horizontal arrows, $Y_{i}$ the arrows pointing up and to the left, and $Z_{i}$ the arrows pointing down and to the left. For notational simplicity, we shall often write $X_{0} \equiv Z_{1}$ interchangeably. We denote by lowercase letters the corresponding R-charges. Owing to the $\mathbb{Z}_{2}$ symmetry of the quiver, we have:

$$
x_{i}=x_{n+1-i}, \quad y_{i}=z_{n+1-i}, \quad \text { and } \quad z_{i}=y_{n+1-i} .
$$


The trial $a$-function is given by:

$$
a_{\text {trial }}=\frac{3 N^{2}}{32}\left(2(n-1)+\sum_{i=1}^{n}\left(f\left(x_{i}\right)+f\left(y_{i}\right)+f\left(z_{i}\right)\right)\right) \text {, }
$$

where $f(x) \equiv 3(x-1)^{3}-(x-1)$. The overall constant is due to the contribution from the gauge group factors, and the factor of $N^{2}$ is due to the fact that each field in the UV is an $N \times N$ matrix. To perform $a$-maximization, we first impose the beta function and superpotential conditions of equations (3.4) and (3.5), respectively:

$$
\begin{aligned}
x_{i+1}+x_{i}+y_{i+1}+z_{i} & =2, \\
x_{i}+y_{i}+z_{i} & =2,
\end{aligned}
$$

for $i=1, \ldots, n$. Here, the lowercase letter denotes the R-charge of the field. Solving in terms of the $x_{i}$ yields:

$$
y_{i}=2-\sum_{j=0}^{i} x_{j} \quad \text { and } \quad z_{i}=\sum_{j=0}^{i-1} x_{j} .
$$

We therefore need to perform $a$-maximization over the $n+1$ independent parameters $x_{0}, \ldots, x_{n}$. To simplify $a_{\text {trial }}[x]$, we use the fact that $f(2-x)=-f(x)$. After some algebraic manipulation, one finds that the trial $a$-function considerably simplifies to:

$$
a_{\text {trial }}[x]=\frac{3 N^{2}}{32}\left(2(n-1)-f\left(x_{\Sigma}\right)+\sum_{i=0}^{n} f\left(x_{i}\right)\right),
$$

where we have introduced $x_{\Sigma} \equiv x_{0}+\ldots+x_{n}$. The local maximum has all $x_{i}$ equal, and given by:

$$
x_{i}=\frac{2}{n+2} .
$$

An important subtlety with this analysis is that it does not take into account the possibility of accidental $U(1)$ symmetries in the infrared. That such symmetries will appear can be seen from examining the R-charge assignments for composite operators which we refer to as mesons:

$$
\begin{aligned}
& \mathcal{M}_{j}=X_{1} \ldots X_{j} Z_{j}, \\
& \mathcal{M}_{j}^{\prime}=Y_{n-j+1} X_{n-j+1} \ldots X_{n},
\end{aligned}
$$

Using the R-charge assignments of equation (3.12), one might conclude that the $j^{\text {th }}$ meson has R-charge $4 j /(n+2)$. In other words, for $j$ sufficiently small and $n$ sufficiently large, there can be an apparent violation of the unitarity bound. Now, although the mesons $\mathcal{M}_{1}$ 


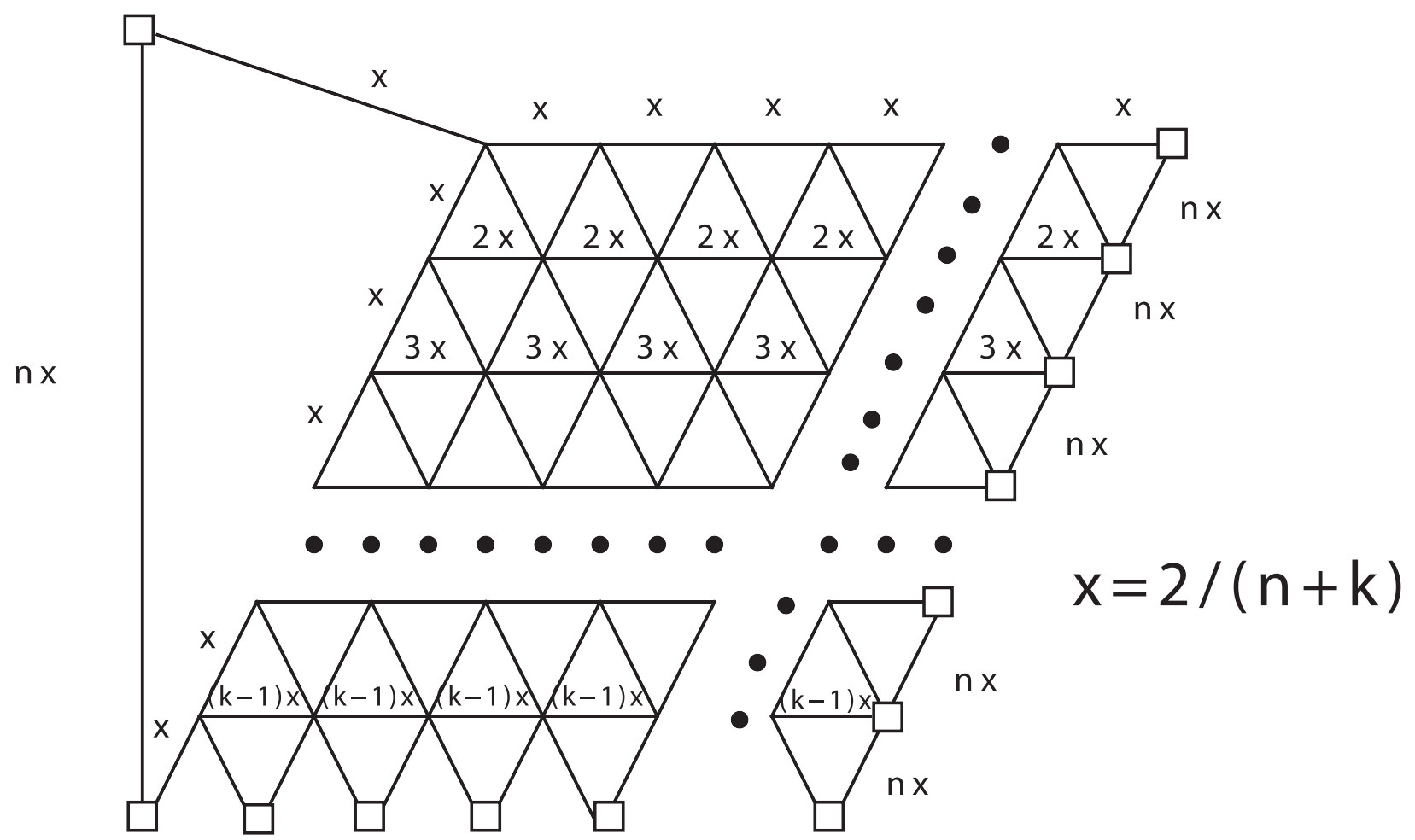

Figure 6: Applying a-maximization without taking into account the decoupling of mesons, we can compute the "naive" R-charge assignments for the bifundamentals. Here we indicate the conjectured R-charges for the top cell of $\mathrm{Gr}_{k, n+k}^{\mathrm{tnn}}$. At sufficiently large $k$ and $n$, these values will be corrected due to the appearance of accidental $U(1)$ 's in the IR theory. To avoid clutter, we only indicate some of the R-charges; the remaining ones follow from equations (3.4) and (3.5). All of the R-charges are proportional to $x=2 /(n+k)$.

and $\mathcal{M}_{1}^{\prime}$ have R-charge below $2 / 3$ when $n>4$, we observe that in the chiral ring they are set to zero, so there is no issue with a violation of the unitarity bound for these operators. By contrast, for $j \geq 2$, there could indeed be an apparent violation of the unitarity bound. This does not signal an inconsistency of the theory, but rather, provides a strong indication for an accidental $U(1)$ in the infrared.

Following the procedure of [12 14, we can postulate the existence of an extra $U(1)$ which acts only on the "offending" operator so that it can decouple as a free field as it hits the unitarity bound. Including a sequence of such extra $U(1)$ 's (as each further meson decouples), the form of the trial a-function is now:

$$
a_{\text {new }}[x]=a_{\text {old }}[x]+\frac{3 N^{2}}{32}\left(\frac{2(r-1)}{9}+\frac{2(r-1)}{9}\right)-a_{\text {left }}^{(r)}[x]-a_{\text {right }}^{(r)}[x],
$$


where the subtracted contribution from the mesons is:

$$
\begin{gathered}
a_{\text {left }}^{(r)}[x]=\frac{3 N^{2}}{32} \sum_{j=2}^{r} f\left(z_{1}+x_{1}+\ldots+x_{j}\right), \\
a_{\text {right }}^{(r)}[x]=\frac{3 N^{2}}{32} \sum_{j=1}^{r-1} f\left(y_{n}+x_{n}+\ldots+x_{n-j}\right) .
\end{gathered}
$$

Extremizing $a_{\text {new }}[x]$, the R-charges now organize according to exterior regions, for $0 \leq i \leq r$ or $n-r+1 \leq i \leq n$ and an interior region, for $r<i<n-r$. All of the R-charges for the interior $X_{i}$ are identical, while the R-charges for the exterior $X_{i}$ obey a sequence of nested square root functions. A full analysis of these solutions is beyond the scope of the present paper. As should be clear, these theories exhibit a surprisingly rich phase structure which deserves further study.

Similar considerations apply in the $\operatorname{Gr}_{k, n+k}^{\mathrm{tnn}}$ theories with $k>2$. In figure 6 we present a conjecture for the "naive" R-charge assignments of the theory. As $k$ and $n$ grow, however, we can expect mesons to decouple, which will in turn modify the computation of the infrared R-symmetry, leading to a gradation of R-charge assignments as one passes from the outside of the graph to the interior. Qualitatively this is similar to a variational problem for the area of a two-dimensional membrane.

\section{Bipartite Graphs and $\mathcal{N}=2$ BPS Quivers}

Although our main interest in this paper is the $\mathcal{N}=1$ theory defined by a given bipartite graph, much of the underlying geometric structure borrows from the string construction of generalized Argyres-Douglas $(\mathrm{AD}) \mathcal{N}=2$ theories. In preparation for our stringy realization of the $\mathcal{N}=1$ bipartite theories, in this section we show that much of this structure is also present in $\mathcal{N}=2$ theories, and in particular the BPS quiver associated with a given theory.

The BPS quiver is a convenient way to capture data about $1 / 2$ BPS states of an underlying $\mathcal{N}=2$ theory. In this subsection we explain how these quivers are related to our bipartite graphs. The main statement is that in an appropriate chamber, the BPS quiver of the generalized $\mathrm{AD}$ theory of type $\left(A_{k-1}, A_{n-1}\right)$ realizes the internal nodes of the bipartite theories associated to $\operatorname{Gr}_{k, n+k}^{t n n}$. Most of the observations we make in this section are wellknown to experts and can be found for example in [15 18]. See also [19] for some discussion of the relation between bipartite graphs and spectral networks. The main distinction from the $\mathcal{N}=2$ case is the absence of flavor symmetries for the planar bipartite graphs. This will automatically appear in our stringy realization of the $\mathcal{N}=1$ theories.

The $\mathcal{N}=2$ generalized $\left(A_{k-1}, A_{n-1}\right)$ AD theories can be obtained in a number of ways from string theory. They arise from compactification of type IIB strings on the singular 
Calabi-Yau threefold $y^{k}=x^{n}+u v$ (see e.g. [15, 20]), which in the mirror IIA geometry is characterized by an NS5-brane wrapped on the singular algebraic curve:

$$
f(x, y)=y^{k}-x^{n}
$$

in $\mathbb{C}^{2}$, with holomorphic coordinates $x$ and $y$. In the M-theory lift this corresponds to an M5-brane wrapped on the same curve. Throughout we refer to this algebraic curve and its deformations as $\Sigma$.

The Coulomb branch of the $\left(A_{k-1}, A_{n-1}\right)$ AD theory corresponds in the geometry to switching on specific lower order deformations of the form $x^{i} y^{j}$ in the geometry. The charge lattice of this theory has dimension $(k-1)(n-1) 6$ A $1 / 2$ BPS state is specified by a choice of $\mathcal{N}=1$ subalgebra of the $\mathcal{N}=2$ theory, corresponding (in the M5-brane realization of the theory) to M2-branes wrapped over two-chains ending on homology 1-cycles of $\Sigma$. The $1 / 2$ BPS states of this $\mathcal{N}=2$ theory are encoded in BPS flow lines, and this data can in turn be packaged in terms of a BPS quiver.

In the BPS flow line picture (see [20]), one tracks the constant phase trajectories of the Seiberg-Witten differential $\lambda_{S W}=y d x$. This is locally specified by the flow equation:

$$
y d x=\alpha d t
$$

for $\alpha=\exp (i \theta)$ a constant phase which specifies a choice of $\mathcal{N}=1$ subalgebra. Such flows meet at the ramification points of $\Sigma$, viewed as a $k$-sheeted cover over the $x$-plane. A homology 1-cycle which encircles two such ramification points specifies a BPS state of the theory. This projection to the $x$-plane is equivalently characterized by the spectral network of the theory [18].

The spectrum of $1 / 2$ BPS states of the generalized AD theory is also encoded in the BPS quiver of the $\mathcal{N}=2$ theory [15, 16]. This is just the quiver diagram we would get from characterizing possible bound states of branes in the singular Calabi-Yau $y^{k}=x^{n}+u v$. Depending on the details of the deformations of the singularity, the connectivity of the quiver can be different, corresponding to a mutation i.e. a Seiberg-like duality of the quiver.

The canonical chamber for the BPS quiver of the $\left(A_{k-1}, A_{n-1}\right)$ theory is a $(k-1) \times(n-1)$ rectangular grid of quiver nodes in which each interior node supports two horizontal arrows both pointing into or out of the node, and two vertical arrows anti-correlated with the orientation of the horizontal arrows (see figure 7). By tuning the deformation parameters of $\Sigma$, all of the BPS states can be made mutually supersymmetric. Then, the quiver literally describes the BPS states interpolating between ramification points of $\Sigma$. Note also that the resulting quiver can be described as a bipartite graph with a grid of square faces. Even so,

\footnotetext{
${ }^{6}$ The dimension of the charge lattice is equal to $2 n_{r}+n_{f}$, where $n_{r}$ is the dimension of the $\mathcal{N}=2$ Coulomb branch and $n_{f}$ is the number of $\mathcal{N}=2$ mass parameters.
} 


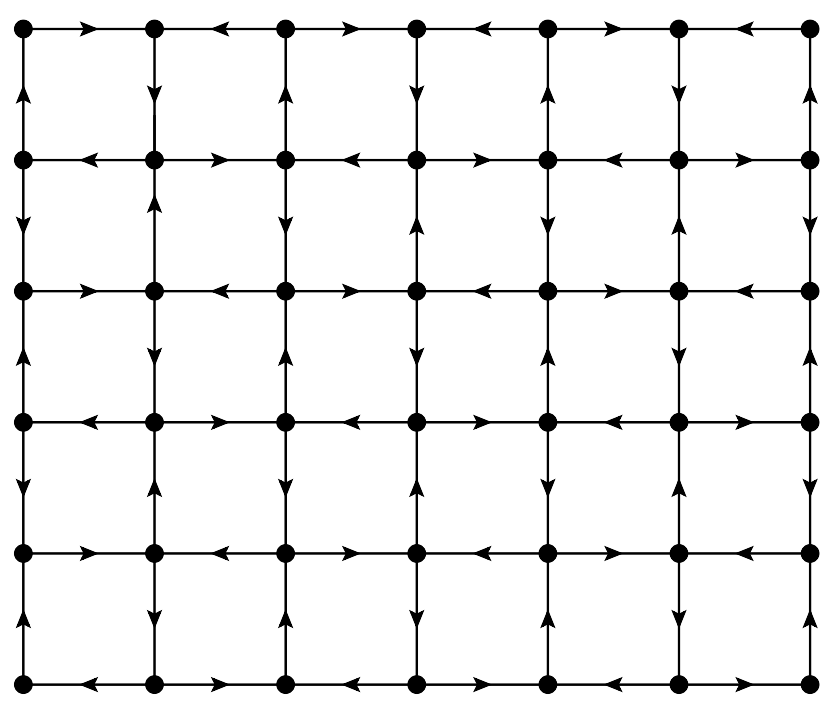

Figure 7: A BPS quiver for the $\left(A_{k-1}, A_{n-1}\right)$ Argyres-Douglas theory. This is given by a $(k-1) \times(n-1)$ grid of nodes, which are attached as in the figure. The rectangular grid of squares specifies the canonical chamber of the theory. Other chambers can be reached by a sequence of mutations (see figure 8).
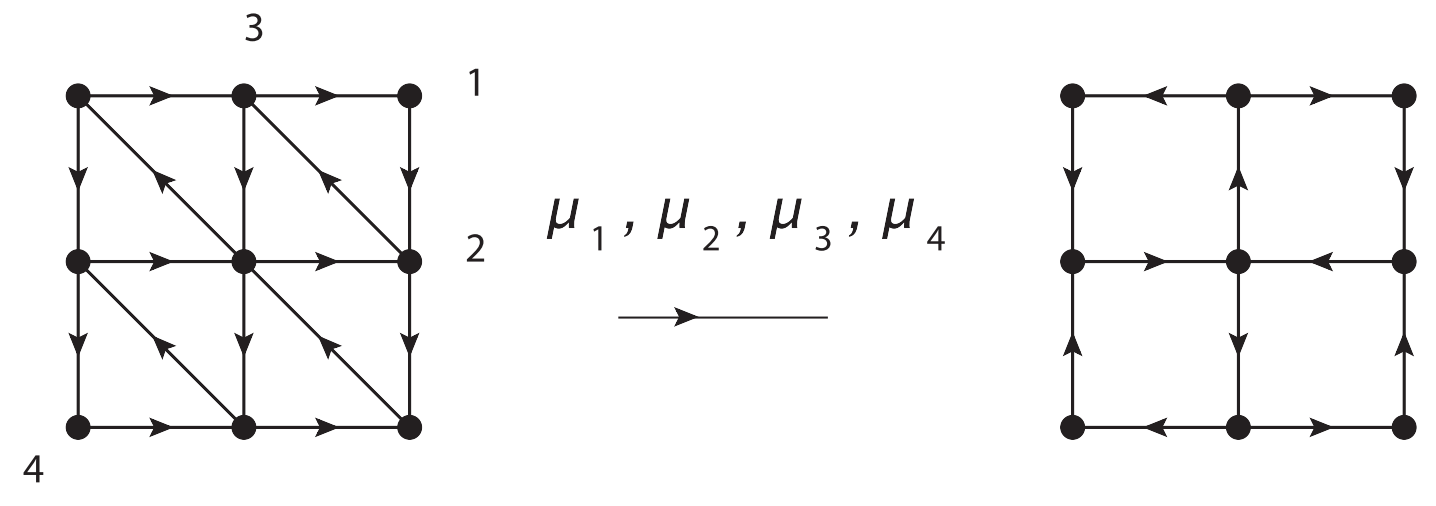

Figure 8: By a sequence of mutations, the internal nodes of the quiver from figure 4 for the top cell of the $\mathrm{Gr}_{k, n+k}^{\mathrm{tnn}}$ theories can be brought to the canonical chamber of the $\mathcal{N}=2$ $\left(A_{k-1}, A_{n-1}\right)$ BPS quiver. In the figure we present this transformation for $k=4$ and $n=4$ by mutation on the nodes labelled $1, \ldots, 4$.

a given mutation of the quiver may not be characterized by a bipartite graph. One can also show that the interior pattern of tiled hexagons depicted in figure 4 can indeed be reached by a sequence of such mutations, see figure 8 for an example. With a little bit of patience, one can see that this is true for general $k$ and $n$.

Higgsing in the BPS quiver corresponds to a partial resolution of the singular geometry, which in the quiver amounts to switching on FI parameters. This in turn triggers non-zero bifundamental VEVs which in the bipartite graph corresponds to deleting the neighboring 

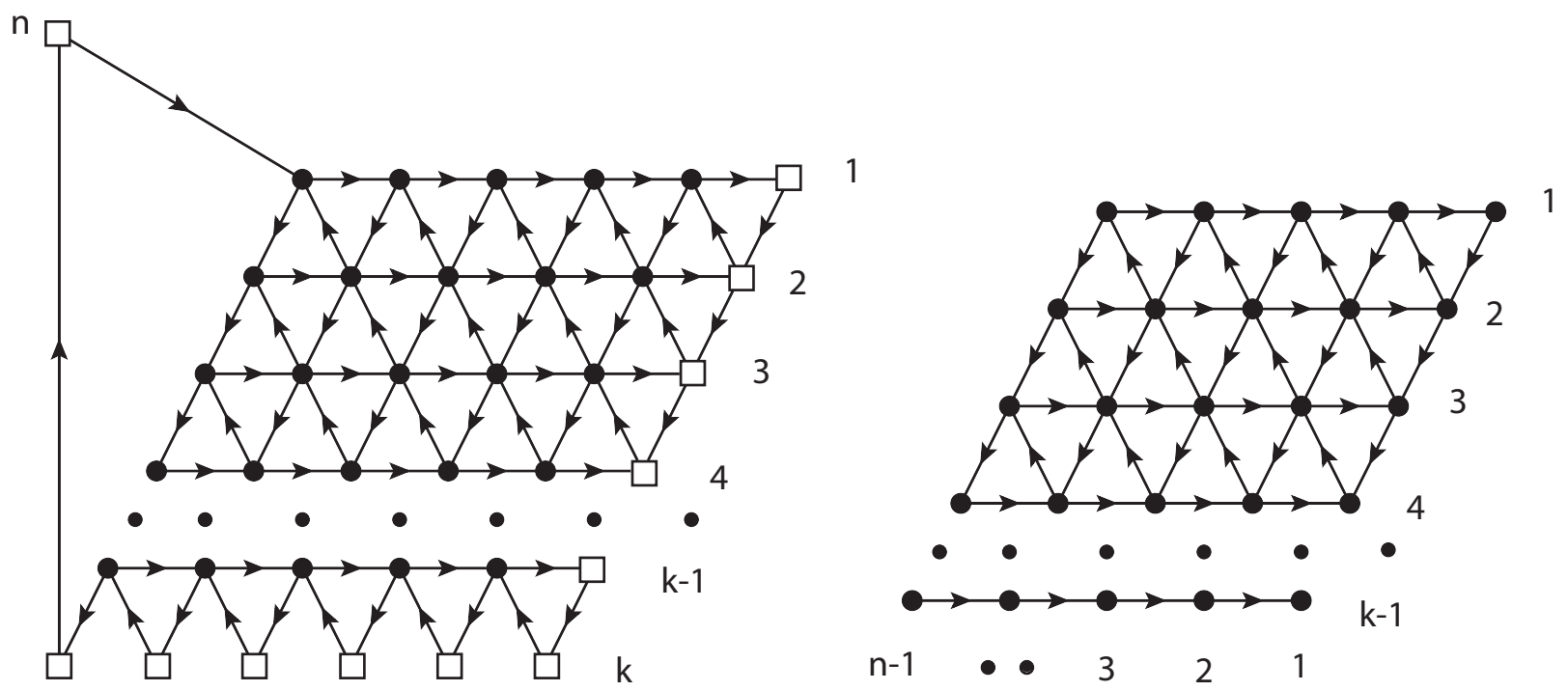

$\mathrm{n}-1$ • $\quad \begin{array}{llll} & 3 & 2 & 1\end{array}$

Figure 9: Comparison of the $4 \mathrm{D} \mathcal{N}=1$ quiver (left) and the BPS quiver for the $4 \mathrm{D} \mathcal{N}=2$ Argyres-Douglas theory (right). The main difference between these two quivers is that the one for the $\mathcal{N}=2$ theory does not contain any flavor nodes, which we denote by squares.

edge between faces (see e.g. [3] for the related statement in $\mathcal{N}=1$ theories). Performing several such Higgsing operations in tandem with mutations, one can see that other bipartite BPS quivers can also be reached.

Summarizing, we see that the interior of a bipartite graph is indeed naturally reproduced from an $\mathcal{N}=2$ BPS quiver. However, we can also identify some differences. If we had tried to interpret the BPS quiver as a 4D gauge theory, there would not be any flavor nodes (see figure 9) and we would find that the gauge theory is either anomalous, or develops a mass gap. Hence, a proper treatment of the flavor groups is essential in the definition of these theories. We now turn to a stringy realization of the $\mathcal{N}=1$ bipartite theories and show how the flavor branes naturally appear.

\section{$5 \quad$ Stringy Realization}

In this section we propose a string theory realization for the bipartite gauge theories introduced in section 2. The basic setup consists of a configuration of intersecting D5-branes and NS5-branes wrapped on mutually supersymmetric two-cycles in the internal geometry. Although the resulting system only preserves $\mathcal{N}=1$ supersymmetry, it turns out that much of the necessary geometric data can be formulated in terms of closely related objects in a corresponding $\mathcal{N}=2 \mathrm{AD}$ theory. The basic idea will be to consider a configuration of in- 
tersections between D5-branes and NS5-branes which are characterized by BPS flow lines of an associated $\mathcal{N}=2$ theory.

Our setup is as follows. We take type IIB string theory on $\mathbb{R}^{3,1} \times \mathbb{C}^{2} \times \mathbb{C}_{\perp}$, with D5branes and NS5-branes filling the four spacetime directions, and wrapping two-dimensional subspaces in the internal geometry. All branes sit at the same point of $\mathbb{C}_{\perp}$. With respect to this fixed complex structure for $\mathbb{C}^{2}$, we take $N$ D5-branes wrapping an algebraic curve $\Sigma \subset \mathbb{C}^{2}$ with specified boundary conditions at infinity in $\mathbb{C}^{2}$. In addition to these D5-branes, we consider NS5-branes wrapped on non-compact special Lagrangian submanifolds inside of $\mathbb{C}^{2}$ which intersect the D5-branes along 1-cycles of $\Sigma$. These intersections serve to partition the D5-brane into independent pieces. Indeed, once the NS5-branes have been added, the D5-branes can be viewed as separate branes which are suspended between the NS5-branes. In this configuration, there are moduli associated with the VEVs of massless bifundamentals stretched between the various D5-branes. These directions in the moduli space correspond to the motion of the distinct suspended D5-branes in the configuration.

The algebraic curve $\Sigma$ wrapped by the D5-branes is the zero set $f(x, y)=0$ with:

$$
f(x, y)=y^{k}-x^{n}+\sum a_{i j} x^{i} y^{j}
$$

where $x$ and $y$ are holomorphic coordinates of $\mathbb{C}^{2}$ and the terms multiplying $a_{i j}$ are lower degree deformations. Note that in the absence of the NS5-branes, the D5-branes would preserve $\mathcal{N}=2$ supersymmetry. In contrast to an M5-brane wrapped over the geometry with all deformations switched off, the resulting IR theory produced by the D5-brane would not describe an interacting fixed point. The reason is that Euclidean D1-branes wrapped on two-chains ending on one-cycles will in general smooth out the singular behavior of the classical geometry (see e.g. [21]). Compactifying on a further circle, however, leads to a three-dimensional theory which is identical to the circle compactification of the $\mathcal{N}=2$ $\left(A_{k-1}, A_{n-1}\right)$ AD theory.

Adding NS5-branes wrapped on mutually supersymmetric special Lagrangians breaks the supersymmetry further, leaving us with a $4 \mathrm{D} \mathcal{N}=1$ theory. This choice of an $\mathcal{N}=1$ subalgebra has a close cousin in the related $\mathcal{N}=2$ theories: It specifies BPS flow lines of the generalized AD theory. In the case at hand, a network of BPS flows determines a set of special Lagrangian submanifolds in $\mathbb{C}^{2}$ which are wrapped by NS5-branes. Recall that a special Lagrangian is specified by the conditions:

$$
\left.J\right|_{L}=0 \text { and }\left.\operatorname{Im} e^{-i \theta} \Omega\right|_{L}=0
$$

where $J=\frac{i}{2}(d x \wedge d \bar{x}+d y \wedge d \bar{y})$ denotes the Kähler form of $\mathbb{C}^{2}$ and $\Omega=d x \wedge d y$ is the holomorphic two-form. The parameter $\theta$ specifies a choice of $\mathcal{N}=1$ subalgebra which is preserved. Integrating the holomorphic two-form over a contour normal to $\Sigma$ yields the Seiberg-Witten 
differential $\lambda_{S W}=y d x$. Given such a phase $\alpha=\exp (i \theta)$, there is a corresponding BPS flow line satisfying the differential equation:

$$
y d x=\alpha d t .
$$

Conversely, given such a BPS flow line, there exists a special Lagrangian in $\mathbb{C}^{2}$ which intersects $\Sigma$ along this flow line. See Appendix B for how to reconstruct a special Lagrangian in $\mathbb{C}^{2}$ given a BPS flow line on $\Sigma$.

Partitioning the D5-brane up into distinct components realizes an $\mathcal{N}=1$ quiver gauge theory. Each disconnected component on a sheet of $\Sigma$ specifies either a flavor group, or a gauge group. More precisely, to maintain dynamical gauge groups we assume that at large $x$ and $y$ the geometry has been cut off. Dynamical gauge group factors correspond to at most logarithmic running with the cutoff, while flavor groups exhibit power law running, a feature we explain further in subsection 5.3 ,

In addition to these symmetry groups, when two disconnected regions share a onedimensional interface, there will be a bifundamental chiral superfield localized there. When three or more such 1-cycles of the network meet at a point, a worldsheet disk instanton localized at the intersection point generates a superpotential interaction term between the corresponding bifundamentals. This gives rise to a quiver gauge theory, with superpotential interaction terms dictated by the appearance of more than two lines meeting.

As should now be clear, our construction is closely related to the BPS quiver of the $\mathcal{N}=2 \mathrm{AD}$ theories. Indeed, while the interiors of the two quivers are the same, there is an important distinction in the appearance of flavor branes for the $\mathcal{N}=1$ theories. We now explain why the interior of the $\mathcal{N}=1$ quiver gives the BPS quiver for $\mathcal{N}=2$ theories. As we change the phase of the BPS flow lines, the topology of the flow lines change precisely at phases corresponding to where they are given by the phase of the central charge of the $\mathcal{N}=2$ BPS state. Consider the case where we take the parameters of the $\mathcal{N}=2$ curve to give almost the same phase for all the BPS states. In this limit the interior regions collapse to regions with zero area. In this limit the theory one gets for the $\mathcal{N}=1$ quiver, if we $\mathrm{T}$ dualize three of the spatial dimensions and keep only the interior branes is the $\mathcal{N}=2$ BPS quiver 7 . This thus explains why the interior part of the $\mathcal{N}=1$ quiver agrees with that of the $\mathcal{N}=2$ BPS quiver. The geometry of the flavor branes is fixed by the asymptotics of the BPS flow lines at large $x$ (c.f. Appendix B), which partitions a sheet of the cover into $n+k$ distinct asymptotic regions. In other words, combining the asymptotic behavior of the flows with the interior behavior predicted by the BPS quivers, we reconstruct the general pattern of quiver gauge group factors found in the bipartite theories! More precisely, we recover the

\footnotetext{
${ }^{7}$ Note that this also explains why the $\mathcal{N}=1$ theory would have been anomalous if we had just kept the interior regions of the quiver, since T-dualizing all spatial directions would yield a situation where the flux due to BPS charges has nowhere to go.
} 
most generic class of quiver gauge theories, corresponding to the top cell of $\mathrm{Gr}_{k, n+k}^{\mathrm{tnn}}$.

Reaching the lower cells of $\mathrm{Gr}_{k, n+k}^{\mathrm{tnn}}$ is also straightforward in this construction. In the bipartite graph, we reach these cells by deleting some of the internal lines of the bipartite graph. In the quiver gauge theory, deleting a line corresponds to activating a pair of FI parameters on the two faces which share this edge [3]. These FI parameters are given by background values of two-form potentials integrated over appropriate two-chains. Setting these FI parameters to $\zeta$ and $-\zeta$ triggers a $\mathrm{VEV}$ for the bifundamental which is consistent (via earlier results on dimer models) with all F- and D-term constraints. These VEVs then break the two gauge groups to its diagonal via the Higgs mechanism.

In the remainder of this section we discuss in more detail the construction of these quiver gauge theories. To give a set of illustrative examples, in subsection 5.1 we show in detail how our construction reproduces the cells of $\mathrm{Gr}_{2, n+2}^{\mathrm{tnn}}$. This case illustrates the main aspects of the general construction, and also reveals that the white and black vertices attached to external legs are indeed treated differently in our construction. In subsection 5.2 we exploit the geometric connection with related $\mathcal{N}=2$ theories to show how the $\operatorname{Gr}_{k, n+k}^{\mathrm{tnn}}$ theories are reproduced from our construction. This is followed by a more detailed discussion of the cutoff geometry, and the class of admissible deformations of $\Sigma$ in subsection 5.3. Finally, making use of this geometric characterization of our theories, in subsection 5.4 we show that the local combinatorics such as the "square move" (i.e. Seiberg duality) is reproduced by the brane construction. This establishes that the IR fixed points are indeed characterized by the cells of the Grassmannian.

\subsection{The $\mathrm{Gr}_{2, n+2}^{\mathrm{tnn}}$ Theories}

In this subsection we show how our proposal works in more detail in the special case $k=2$ so that the curve $\Sigma$ is given by:

$$
y^{2}=P_{n}(x)=\prod_{i=1}^{n}\left(x-a_{i}\right) .
$$

This is the curve for the $\left(A_{1}, A_{n-1}\right)$ Argyres-Douglas theories. The BPS quiver for these theories consists of a single row of $(n-1)$ nodes. What we are going to show is how our $\mathcal{N}=1$ construction based on BPS flow lines naturally supplements this quiver by additional flow lines. In particular, this will convert the quiver nodes which would have been anomalous in a 4D theory into anomaly free gauge group factors. See figure 10 for a depiction of the $\mathcal{N}=2$ BPS quiver, the $\mathcal{N}=1$ quiver, and the corresponding flow lines and bipartite graph.

Recall that the BPS flow lines of $\Sigma$ are given by constant phase trajectories of $\lambda_{S W}=y d x$. 


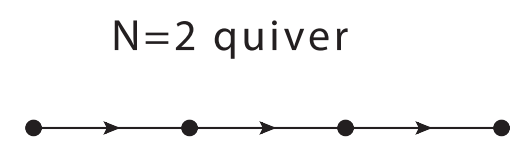

BPS flow

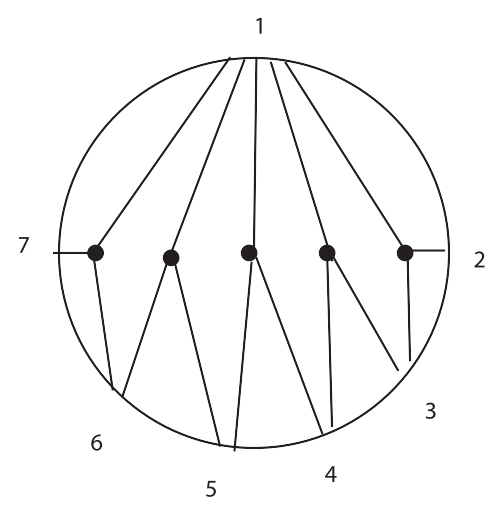

$\mathrm{N}=1$ quiver

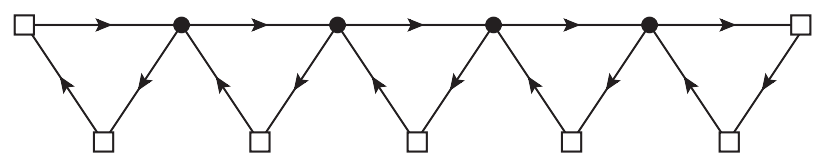

Bipartite network

1

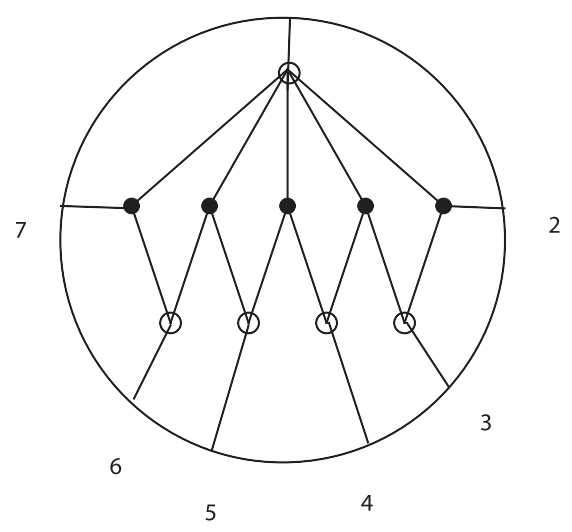

Figure 10: Depiction of the $\left(A_{1}, A_{n-1}\right) \mathcal{N}=2$ BPS quiver, the corresponding $\mathcal{N}=1$ quiver, and the flow lines for theories associated with the top cell of $\mathrm{Gr}_{2, n+2}^{\mathrm{tnn}}$. Additionally, we present the corresponding bipartite network. For specificity, in the figure we indicate the case of $n=5$. See figure 5 for the R-charge assignments of the $\mathcal{N}=1$ theory, and figure 11 for another example of flow lines and the corresponding bipartite network.

Introducing a local parametrization $t$ for the flow, this can locally be written as the condition:

$$
y d x=\alpha d t
$$

for $\alpha=\exp (i \theta)$ a choice of phase for the sLag. This constant phase condition has been studied in the context of BPS flow lines of $\mathcal{N}=2$ systems [20], and also appears in the WKB approximation of the complexified quantum mechanics with Hamiltonian $H(x, p)=$ $p^{2}-P_{n}(x)$ :

\footnotetext{
${ }^{8}$ In the complexified quantum mechanics, the BPS flow lines specify saddle point configurations of the complexified path integral:

$$
\int \mathcal{D}[x(t)] \mathcal{D}[p(t)] \exp \left(i \int p d x-H(x, p) d t\right)
$$

where here, $H(x, p)$ is a general holomorphic polynomial in $x$ and $p$. The complexification is similar in spirit to the discussion in [22]. A level set $H=E$ defines a Riemann surface in the $\mathbb{C}^{2}$ parameterized by $x$ and $p$. A stationary phase of the path integral specifies a complex trajectory in
} 
The asymptotic geometry of the BPS flow lines at large $x$ and $y$ is dominated by the highest degree term $x^{n}$ of $P_{n}(x)$. Integrating the flow equation in this case yields the value of $x$, treated as a function of $t$ :

$$
x^{(n+2) / 2}-x_{0}^{(n+2) / 2}=\beta t,
$$

where $\beta$ is a constant with the same phase as $\alpha$, and $x_{0}$ is the value of $x$ at $t=0$. In other words, the asymptotics of the one-cycle are fully specified by the phase $\exp (i \theta)$. We see that since $t \in \mathbb{R}$, there are precisely $n+2$ asymptotic regions for the one-cycles, given by $x \sim r \zeta_{n+2}$ for $r \gg 0$ and $\zeta_{n+2}$ an $(n+2)$-th root of unity. This specifies a boundary condition for the NS5-brane, which remains fixed once we specify a choice of $\mathcal{N}=1$ algebra.

In the interior of the $x$-plane these asymptotic flows will meet at roots of $P_{n}(x)$. Near a given root of $P_{n}(x)$, we can locally integrate equation (5.5). If the root of $P_{n}(x)$ vanishes to order $m$, this yields the local behavior of the flow near this root:

$$
\left(x-a_{i}\right)^{(m+2) / 2} \simeq \beta^{\prime} t^{\prime}
$$

where $\beta^{\prime}$ is a constant with the same phase as $\alpha$. This locally defines the meeting of $m+2$ BPS flow lines. In particular, we see that in the generic case where $m=1$, this specifies a trivalent vertex.

These trivalent vertices naturally map to vertices of a bipartite graph. Indeed, each region of the $x$-plane divided by the flow lines specifies a gauge group factor, and D5/D5' strings stretched across the interface provide matter fields. Note that since the BPS flow lines come with a choice of local orientation, the GSO projection leads to a chiral superfield with a designated orientation in the quiver gauge theory. Superpotential interactions generated by a worldsheet disk instanton localize at the root $x=a_{i}$. In other words, this reproduces one of the building blocks of the bipartite theories.

But in addition to regions where the BPS flow lines meet at a root, there can also be regions where the flow lines nearly meet. In this situation, two or more BPS flow lines in the interior asymptote to the same root of unity at large $x \sim r \zeta_{n+2}$. Tracing these flows back into the interior, one can see that although they come close to touching, they eventually diverge from one another. Asymptotically, one can still view this as a trivalent vertex, but one in which neighboring non-compact faces do not contribute an additional bifundamental. Consequently, there is also no superpotential interaction term associated with the corresponding vertex. In other words, we see that the BPS flow lines either terminate

the $(x, p)$ phase space which we can denote by $(x(t), p(t))$. In this system, we observe that along a level set, the stationary phase configuration corresponds to the case where $\int p d x$ has a fixed phase along a given trajectory. In other words, the BPS flow line specifies the WKB approximation of the complexified quantum mechanics. 

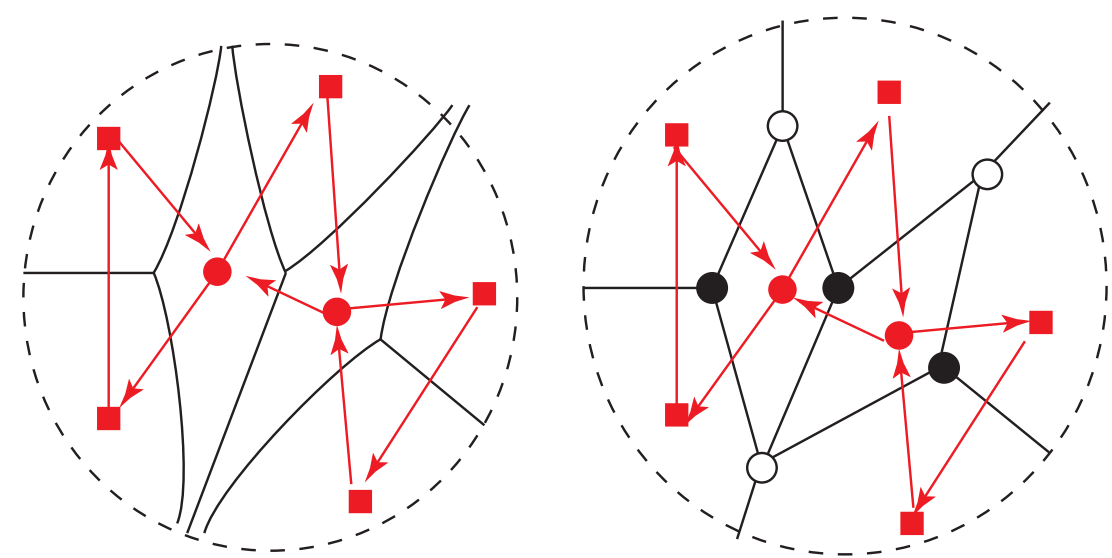

Figure 11: Depiction of the flow lines and corresponding bipartite graph realized by a configuration of D5-branes wrapping $y^{2}=x^{3}+$ (deformations), with NS5-branes intersecting the D5-brane along the BPS flow lines of the related $\mathcal{N}=2\left(A_{1}, A_{2}\right)$ Argyres-Douglas theory.

at trivalent vertices, or form asymptotic flows.

These flows come in two types, and for the class of $y^{2}=P_{n}(x)$ theories reproduce the bipartite graph associated with the top cell of $\mathrm{Gr}_{2, n+2}^{\mathrm{tnn}}$. To see this, order the roots of $P_{n}(x)$ so that $\operatorname{Re} a_{1} \leq \operatorname{Re} a_{2} \leq \ldots \leq \operatorname{Re} a_{n-1} \leq \operatorname{Re} a_{n}$. The trivalent vertices produce a pattern of diamonds which are glued pairwise along a common edge. See figure 11 for a depiction of the flow lines for the case $k=2$ and $n=3$. The generalization to higher $n$ for a particular choice of duality frame is shown in figure 10. The pattern of flow lines maps out a collection of "diamonds" which are glued together to form a single row of squares. Let us note that in general, there is no unique bipartite graph constructed from these flows. For example, by moving the roots along the imaginary axis, we can either produce a row of diamonds which all share a single white vertex, or alternatively, we can produce a row of squares which only share adjacent sides. In any case, the external data is the same: we obtain a bipartite graph with an external leg attached to the leftmost black vertex and another external leg attached to the rightmost black vertex, with the $n$ remaining legs attached to white vertices. In other words, we have found the bipartite graphs associated with the top cell of $\mathrm{Gr}_{2, n+2}^{\mathrm{tnn}}$ ! As already mentioned, we arrive at the lower cells by switching on background values for field-dependent FI parameters. This triggers a brane recombination, corresponding to deleting internal lines of the bipartite graph.

An important feature of our construction is that the flavor group factors are not anomalous which means we are free to weakly gauge these symmetries. This is because the "external lines" associated with the white vertices do not contribute a bifundamental mode. Thus, we conclude that our construction realizes the rules for external legs proposed in [1]. 


\subsection{The $\mathrm{Gr}_{k, n+k}^{\mathrm{tnn}}$ Theories}

In this subsection we show how our construction generalizes to the $\mathrm{Gr}_{k, n+k}^{\mathrm{tnn}}$ theories, corresponding to taking the curve $\Sigma$ associated with the $\left(A_{k-1}, A_{n-1}\right)$ AD theories. In this case, we can view $\Sigma$ as a $k$-sheeted cover over the $x$-plane, with ramification points at the locations where different sheets meet. Throughout, we work in terms of the projection down to the $x$-plane.

The trajectory of the BPS flow lines is again dictated by the condition that $\lambda_{S W}=$ $y d x$ has a constant phase. One important difference from the $k=2$ case is that $y$ has $k$ branches $y_{1}(x), y_{2}(x), \ldots, y_{k}(x)$. Correspondingly we could consider $k(k-1) / 2$ SeibergWitten differentials $\lambda_{i j}:=y_{i} d x-y_{j} d x$, and the same number of flow lines labeled by $(i j)$ :

$$
\lambda_{i j}=\left(y_{i}-y_{j}\right) d x=e^{i \theta} d t
$$

with constant $\theta$. Note that the flow lines come with a natural orientation, such that the value of $\operatorname{Re}\left(e^{-i \theta} \lambda_{i j}\right)$ increases along a flow. Obviously the orientation flips when we exchange $i$ with $j$.

Let us for the moment concentrate on one of the flow lines. Away from the ramification points, we can see that the asymptotics of the BPS flow line picture will be quite similar to the case $k=2$. Integrating the flow equation yields the value of $x$, treated as a function of $t$ :

$$
x^{(n+k) / k}-x_{0}^{(n+k) / k}=\beta t
$$

so that now there are $n+k$ asymptotic regions on the sheet. Continuing this asymptotic behavior into the interior of the $x$-plane, we can see that some of these flow lines will remain as a single line, while others will split apart, corresponding to lines which nearly touch. Just as in the $k=2$ case, these two possibilities correspond to external lines which either attach to a black vertex or a white vertex. This in turn specifies the details of possible bifundamentals connecting the flavor branes.

Going into the interior region, it should be clear that the global structure of the flows will be more involved than in the $k=2$ case. The main physical content we need to track corresponds to the meeting of flow lines near a ramification point of the curve $\Sigma$. This interior structure is in turn dictated by the spectral network of the related $\mathcal{N}=2$ theory [18]. Before turning to this global characterization, let us first discuss the local structure of flows which meet. The generic situation corresponds to the meeting of three BPS flow lines at a ramification point. Near this meeting point, the sheets which appear in the joining operation satisfy the relation:

$$
\lambda_{i j}+\lambda_{j k}=\lambda_{i k} .
$$

Hence, we see that the triple intersection point satisfies the join and split rule for strings: 

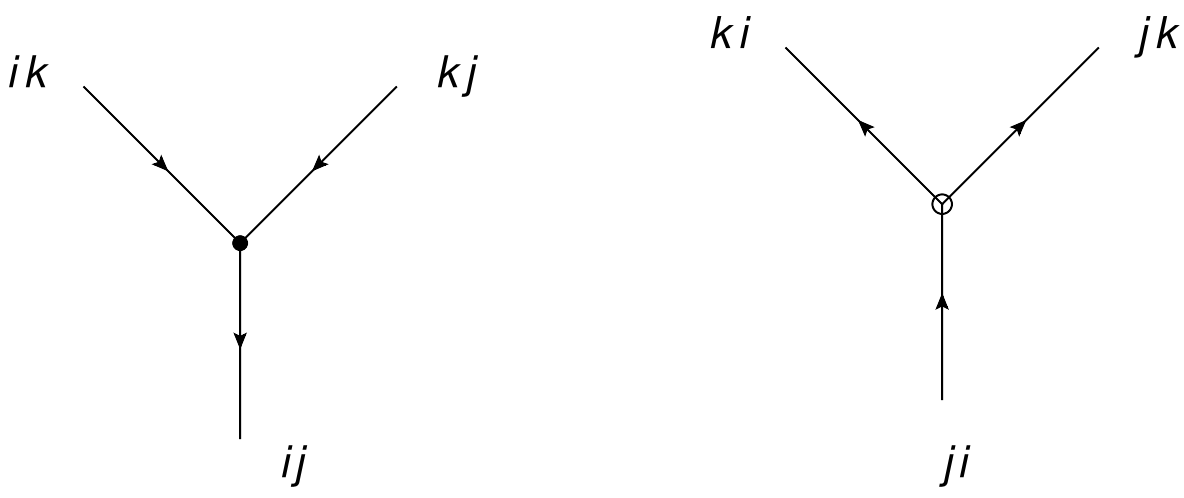

Figure 12: Two kinds of trivalent vertices for the BPS flow lines at the branch points, with different orientations.

$(i j)+(j k) \rightarrow(i k)$. Moreover, there are two possible types of orientations for these flow lines, which we show in figure 12. The reason is that if $(i j)$ and $(j k)$ come into the triple intersection point, then the orientation of $(i k)$ is fixed as outgoing. Alternatively, if $(i j)$ and $(j k)$ are outgoing, then the orientation of $(i k)$ is fixed as incoming. Note that this is the same choice of orientations as in figure 18(a) in Appendix A. This means that the perfect orientation discussed in Appendix A is nothing but the orientation of the flow lines!

Gluing this local data together, we obtain a network of flow lines which meet at the triple intersection points. For this purpose we can combine the trivalent vertices in figure 12 to construct a "skeleton" of the flow lines, as in figure 13. This generates a network which is equipped with a perfect orientation.

12

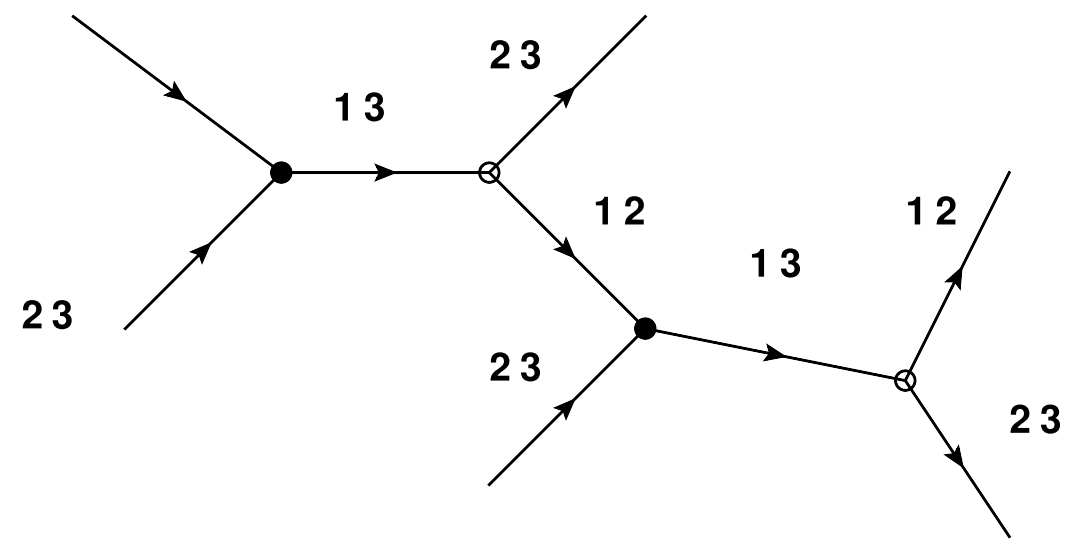

Figure 13: The flow skeleton for the $k=3$ theory.

Let us now come back to the global structure of the flow lines, such as the one given in figure 14. We need to extract the internal connectivity of these theories, and in particular the adjacency of the corresponding quiver gauge theory realized by the network of flow lines. 


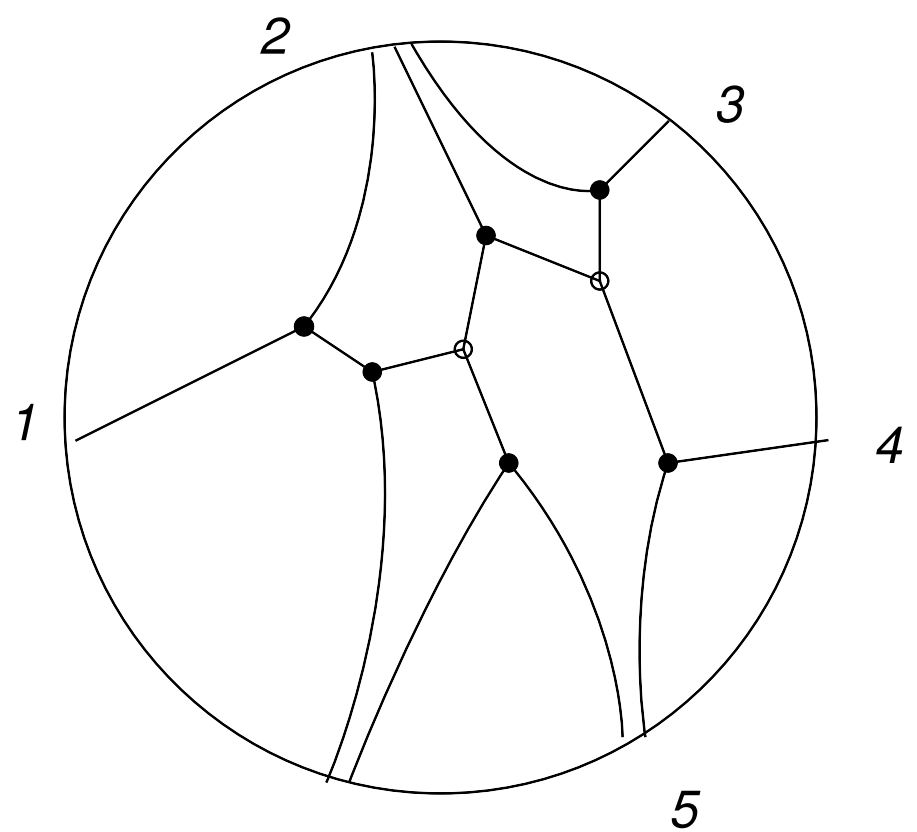

6

Figure 14: An example of the global structure of BPS flow lines, with $k=3, n=3$.

Specifying how all of the sheets glue together more globally is clearly more challenging, as it involves the construction of the spectral network for the general $\left(A_{k-1}, A_{n-1}\right)$ ArgyresDouglas theory. We leave a direct construction of this type for future work. Instead, we follow a different route to extract the corresponding quiver gauge theory specified by the flow lines.

Rather than perform a direct construction, we can combine our knowledge of the asymptotic behavior of the BPS flows with the known internal structure of the quiver to extract the corresponding gauge theories. Indeed, what makes the analysis tractable is the fact that the BPS quivers for the $\left(A_{k-1}, A_{n-1}\right)$ theories are already known [15]. In other words, we already know the internal structure of the quiver gauge theory we are going to get. Further, since we also know that the asymptotic flows terminate on ramification points of the curve $\Sigma$, we can uniquely extend a bipartite chamber of the $\mathcal{N}=2$ BPS quiver to also include flavor branes. Note that this is in accord with the construction we have given for the $\mathrm{Gr}_{2, n+2}^{\mathrm{tnn}}$ theories.

Putting this together, we see that the construction just given realizes the bipartite theories defined by the top cell of $\mathrm{Gr}_{k, n+k}^{\mathrm{tnn}}$. The bipartite graph will also coincide with the skeleton of the flow lines introduced previously. Moreover, as we have already mentioned, we reach the lower cells by a partial Higgsing of the quiver gauge theory. Thus, we see that our construction recovers all of the possible bipartite theories! 
Using the characterization of the interior using the BPS quiver, we can also see that the Grassmannian duality $k \leftrightarrow n$ retains the same connectivity of the bipartite graph, but with the black and white vertices interchanged. The asymptotic flow lines, however, will be different. Indeed, though the number of flavor branes remains the same, we can also see that the bifundamentals connecting them will be distinct. This is in accord with the fact that

in the bipartite theories of [1], the theories for $\mathrm{Gr}_{k, n+k}^{\mathrm{tnn}}$ and $\mathrm{Gr}_{n, n+k}^{\mathrm{tnn}}$ correspond to different fixed points.

\subsection{UV Boundary Conditions}

So far we have shown that the bipartite graphs associated with $\mathrm{Gr}_{k, n+k}^{\mathrm{tnn}}$ are naturally reproduced by the configuration of BPS flow lines for the $\left(A_{k-1}, A_{n-1}\right)$ AD theories. From the structure of the BPS flow lines we can also see, however, that the region enclosed by a "compact" face of the bipartite graph can actually be non-compact (see e.g. figures 10 and 11). Further, since the normalization of the D-term $Q^{\dagger} Q$ for a bifundamental $Q$ is proportional to the length of the corresponding edge, we also see that bifundamentals which are localized along flow lines may also be non-dynamical.

To realize a dynamical quiver gauge theory, it is therefore necessary to cut off our noncompact geometry which in turn specifies a choice of UV boundary conditions for the brane configuration. The basic idea is to evaluate the area enclosed by a region partitioned by the BPS flow lines, using the restriction of the Kähler form:

$$
J=\frac{i}{2}(d x \wedge d \bar{x}+d y \wedge d \bar{y}) .
$$

In the large $x$ regime on the $x$-plane, the contribution from $d y \wedge d \bar{y}$ is subleading, so it is enough to compute the area cut out in the $x$-plane by the BPS flow line.

We now illustrate how to estimate this area and the dependence on a choice of cutoff in the case of the square dimer, specified by the curve $y^{2}=x^{2}-a^{2}$ for real $a>0$. In the large $x$ limit, the asymptotic behavior of $y$ is:

$$
y=\sqrt{x^{2}-a^{2}} \simeq x-\frac{a^{2}}{2 x} .
$$

Integrating this, the constant phase trajectories satisfy:

$$
x^{2}-a^{2} \log x \simeq \beta t
$$

for some constant $\beta$ with the same phase as $\alpha$, and flow coordinate $t$. The asymptotic regime 
$x_{2} \rightarrow \infty$ and $x_{1} \rightarrow 0$ for $x=x_{1}+i x_{2}$ imposes the condition:

$$
x_{2} \simeq \frac{4}{\pi a^{2}} \frac{1}{x_{1}} .
$$

In other words, the area underneath the curve $x_{2}\left(x_{1}\right)$, viewed as a function of $x_{1}$ depends logarithmically on the cutoff:

$$
\int_{\varepsilon} x_{2}\left(x_{1}\right) d x_{1} \simeq-\frac{4}{\pi a^{2}} \log \varepsilon .
$$

This logarithmic running reflects the dependence of $1 / g_{\mathrm{YM}}^{2} \sim$ Area(Face) on the cutoff.

Similar considerations apply for the $\left(A_{k-1}, A_{n-1}\right)$ theories and their deformations:

$$
y^{k}-x^{n}+\sum a_{i j} x^{i} y^{j}=0
$$

In this case, the admissible deformations are those which only alter the UV boundary conditions by at most logarithmically divergent terms. For example in the case $k=2$, deformations which induce at most logarithmic divergences in the area correspond to degree $(n-2) / 2$ or lower terms in $x$. More generally, these lower order deformations correspond in the $\mathcal{N}=2$ theory to all deformations on the Coulomb branch, and the lowest dimension mass deformation.

Including this cutoff dependence, it follows that the compact faces of the bipartite graph correspond to dynamical gauge group factors in the 4D theory. By contrast, we also see that the area of the flavor branes always diverges as a power law in the cutoff. In particular, this means that we indeed have realized the UV definitions of these quiver gauge theories.

It is also of interest to study the bifundamentals connecting flavor branes. Once we introduce the cutoff, this can be viewed in the bipartite graph as attaching an additional white node along each external leg which attaches to a black vertex, and an additional black node for each external leg which attaches to a white vertex. In particular, this shows that the asymptotic white vertices can be viewed as also having an additional vector-like pair of states, that is, massive string modes connecting the two adjacent flavor branes. See figure 15 for a depiction of this operation.

\subsection{Reproducing the Square Move}

Having shown that the bipartite gauge theories are correctly reproduced by our construction, we can now ask whether the various combinatorial moves used to classify possible IR fixed points are also reproduced. Most of the combinatorial equivalences between different bipartite graphs follow from statements in the corresponding weakly coupled gauge theory. For 


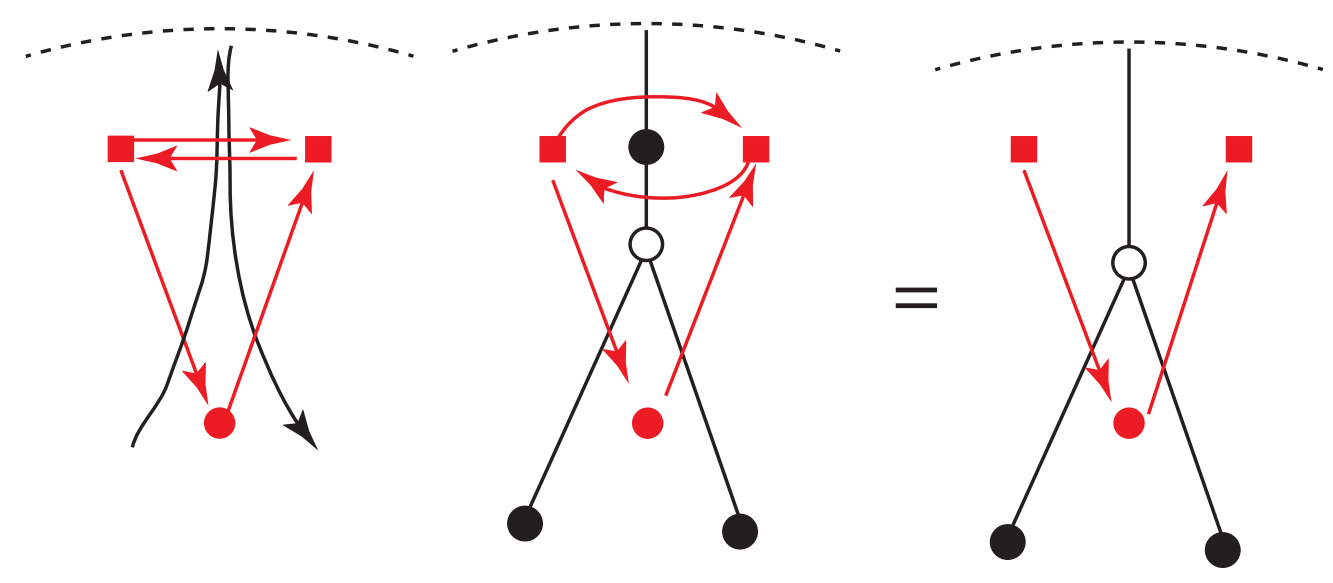

Figure 15: Cutting off the asymptotic behavior of the flows specifies UV boundary conditions for the field theory. This in turn makes the gauge groups and some of the bifundamentals dynamical. Graphically, this cutting off procedure can be viewed as attaching a black node to each external leg which attaches to a white node. This gives a massive vector-like pair of bifundamentals, which in our construction is automatically integrated out.

example, in a bipartite graph with a node attached to only two lines, there is a corresponding mass term available. These modes form a vector-like pair, and are generically massive in the brane construction. In other words, they are automatically integrated out.

The main case which is not apparent from the UV description of the weakly coupled field theory is the "square move", corresponding to the interchange of the black and white vertices, depicted in figure 2(d). In the quiver gauge theory, this corresponds to a Seiberg duality operation. We now show how to reproduce the square move from the BPS flow line picture.

Much as in similar constructions, Seiberg duality of the field theory corresponds in the brane configuration to a change of basis for the branes which realize a given quiver gauge theory. In other words, holding fixed the asymptotics of the intersecting branes, we consider lower order deformations of the geometry. Since the net brane charge is fixed at infinity, the infrared physics is the same. The realization of the quiver gauge theory could, however, be different.

The duality is achieved by varying the location of ramification points on the $x$-plane. Under a generic variation, the geometry of the D5-brane remains smooth, but the geometry of the NS5-branes can jump. As we show in Appendix B, given a BPS flow line, there is a corresponding sLag which we can construct. So in other words, tracking the change of the BPS flow lines is enough to study the possible jumping behavior of the sLags. In the BPS quiver, this is simply a mutation of the quiver, corresponding to moving to a different chamber of BPS states.

Since this jumping behavior of the flow lines is a local phenomenon, it is enough to 

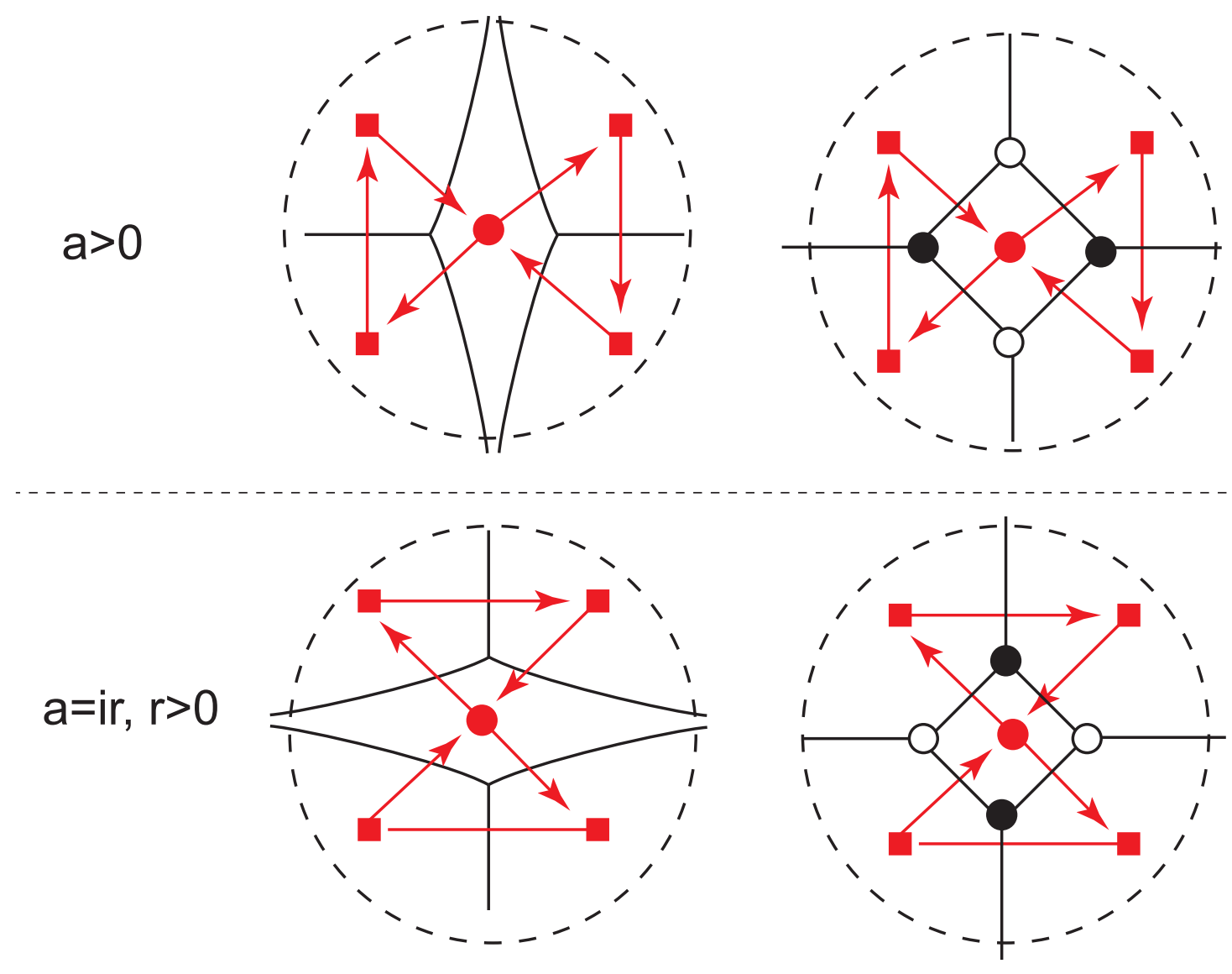

Figure 16: Seiberg duality in the brane construction corresponds to a lower order deformation of the algebraic curve $\Sigma$. Here we depict this operation for the curve $y^{2}=x^{2}-a^{2}$ as a function of the parameter $a$. When we change $a$ from real $a>0$ to imaginary $a=i r, r>0$, the flow lines of the curve $y^{2}=x^{2}-a^{2}$ are rotated by 90 degrees, which is nothing but the square move of the bipartite network.

consider the case $y^{2}=x^{2}-a^{2}$, corresponding to the top cell of $\mathrm{Gr}_{2,4}^{\mathrm{tnn}}$, the single square dimer. When $a>0$, the six BPS flow lines consist of two segments along the real $x$-axis, emanating from $x=a$ to $x \rightarrow \infty$ and $x=-a$ to $x \rightarrow-\infty$. In addition, there are four segments meeting these roots, which emanate out in pairs to either $x \rightarrow i \infty$ or $x \rightarrow-i \infty$. As we rotate $a$ through the complex plane, the asymptotics remain unchanged, but the internal connectivity of the BPS flow lines will change. For example, when $a=i r$ with $r>0$, the asymptotics remain the same, but internally, the entire flow picture has rotated by 90 degrees. Applying our previous rules for extracting a quiver gauge theory, this corresponds to interchanging the black and white vertices of the bipartite graph. See figure 16 for a depiction of Seiberg duality in the geometry.

In the process of such deformations, one can also generically expect a brane creation phenomenon. Note, however, that since the D5-branes are "passive", i.e., they do not ex- 
perience any jumping, it should not be surprising that the rank of the gauge groups in the Seiberg dual has not changed. Indeed, anomaly cancellation of the quiver gauge theory and its Seiberg dual corresponds in the brane configuration to the cancellation of RR and NS tadpoles. Let us note that in the more general possibly non-conformal case where we allow different ranks on the various gauge group factors, there can be a shift in the ranks of a given gauge group factor.

Let us summarize our discussion. We have seen that all of the local combinatorial rules of the bipartite quiver gauge theories are correctly reproduced by our string construction. Hence, the string construction provides a simple explanation for why different quiver gauge theories flow to the same interacting fixed point.

\section{Physical Origin of Coordinates for $\mathbf{G r}_{k, n+k}^{\mathrm{tnn}}$}

In the previous section we showed how our string construction explains the classification of possible IR fixed points by cells of $\mathrm{Gr}_{k, n+k}^{\mathrm{tnn}}$. In this section we show that the coordinates of the Grassmannian are also captured by physical data of our theory. We present evidence that once we compactify our theory, VEVs of line operators in the 3D theory provide a canonical basis of coordinates for $\mathrm{Gr}_{k, n+k}^{\mathrm{tnn}}$. The link we establish is based on the mapping of weight assignments on faces of the bipartite graph to coordinates of $\mathrm{Gr}_{k, n+k}^{\mathrm{tnn}}$, which we review in Appendix A. So in other words, it is enough to give a physical interpretation for the weights of faces in the graph.

The class of objects we consider is dictated by the geometry of the bipartite graph. For each face $f$, there is a canonical choice of surface operator given by a D3-brane suspended between NS5-branes which wraps a two-chain with boundary on the contour of the face and fills two directions of the 4D spacetime. This D3-brane is induced by a gauge field flux in the central $U(1)_{\mathrm{D} 5}$ of a gauge group factor for the D5-brane which fills this same face. Loosely speaking, we associate the VEV of this surface operator $\mathcal{V}_{f}$ with a weight assignment for the face $f$.

To specify the "VEV of a surface operator" in more precise terms, we actually need to compactify our $4 \mathrm{D}$ theory. To this end, we replace our $4 \mathrm{D}$ non-compact geometry by $S_{(t)}^{1} \times M C_{q}$ where $M C_{q}$ denotes the Melvin cigar. The three-dimensional $M C_{q}$ is given by taking a two-dimensional disk $C$ with a cigar-like metric, and fibering it over an additional $S_{(M)}^{1}$, where the coordinate $z$ of $C$ transforms by $z \mapsto q z$ for $q=\exp (i \vartheta)$ a complex phase 9 Our surface operator wraps the $S_{(t)}^{1} \times S_{(M)}^{1}$ factors. To preserve supersymmetry we must twist the theory by embedding the $U(1)_{\mathrm{D} 5}$ in the $U(1)_{R}$ R-symmetry of our superconformal

\footnotetext{
${ }^{9}$ Dimensional reduction of $4 \mathrm{D} \mathcal{N}=1$ theories described by bipartite networks have been discussed recently in 23, 24. Note that the 4D superconformal index discussed there is defined on $S_{(t)}^{1} \times S^{3}$, and the $M C_{q}$ discussed here can be thought of as half of an $S^{3}$ [25].
} 
theory. In the limit where the radius of $S_{(t)}^{1}$ shrinks to zero size, we can T-dualize this circle to the geometry $\widetilde{S}_{(t)}^{1} \times M C_{q}$, obtaining a three-dimensional theory in which the original surface operator is now given by a $U(1)$ holonomy around the circle $S_{(M)}^{1}$. We propose to identify the weight of a face $w_{f}$ with the VEV of the surface operator in the limit where the radius $R_{t}$ of $S_{(t)}^{1}$ tends to zero size:

$$
w_{f}=\lim _{R_{t} \rightarrow 0} \mathcal{V}_{f}
$$

Note that since we are dealing with an $\mathcal{N}=1$ superconformal theory, the resulting $3 \mathrm{D}$ theory will preserve eight real supercharges, four of which will be preserved by the presence of the D3-brane.

To see why the small $S_{(t)}^{1}$ limit is natural and leads to a correspondence between face weights and VEVs of line operators in the 3D theory, first observe that very similar operators appear in the related $4 \mathrm{D} \mathcal{N}=2$ generalized $\left(A_{k-1}, A_{n-1}\right)$ Argyres-Douglas theories. Consider the $4 \mathrm{D} \mathcal{N}=2$ theory obtained from an M5-brane wrapped on the curve $\Sigma$ and filling $\mathbb{R}^{4}$. We can compactify this system on the geometry $\widetilde{S}_{(t)}^{1} \times M C_{q}$. As we have already mentioned, each compact face of the bipartite graph corresponds to a node of the BPS quiver. Though they are not part of the $\mathcal{N}=2$ BPS quiver, we can also include the non-dynamical flavor $U(1)$ factors associated with the $\mathcal{N}=1$ theory. For each such $U(1)$ factor there is a corresponding holonomy, i.e. a choice of line operator which encircles the $S_{(M)}^{1}$. These are 1/2 BPS operators and the choice of $\mathcal{N}=1$ subalgebra is dictated by the R-twisting, i.e. a choice of embedding the $U(1)$ in the $S U(2)_{R}$ R-symmetry of the $\mathcal{N}=2$ theory [15]. A line operator specifies a BPS state, corresponding to an M2-brane which ends on the ramification points of $\Sigma$.

Now in the $\mathcal{N}=2$ setting, the corresponding line operators satisfy the equal time commutator [15]:

$$
\mathcal{U}_{\gamma}(t) \mathcal{U}_{\gamma^{\prime}}(t)=q^{\left\langle\gamma, \gamma^{\prime}\right\rangle} \mathcal{U}_{\gamma^{\prime}}(t) \mathcal{U}_{\gamma}(t)
$$

where the pairing $\left\langle\gamma, \gamma^{\prime}\right\rangle$ is the Dirac-Schwinger-Zwanziger pairing of electric and magnetic states. Moreover, as follows from [15], under a Seiberg-like duality of the BPS flow lines, each such $\mathcal{U}_{\gamma}$ transforms according to the weight transformation rules (in the case $q=1$ ):

$$
w_{f} \rightarrow w_{f}^{\prime}=w_{f}^{-1}, \quad w_{a, c} \rightarrow w_{a, c}^{\prime}=w_{a, c}\left(1+w_{f}\right), \quad w_{b, d} \rightarrow w_{b, d}^{\prime}=w_{b, d}\left(1+w_{f}^{-1}\right)^{-1}
$$

where the boundary of a face is associated with a given contour $\gamma$. Here, the $w_{a, b, c, d}$ denote the weight assignments of the faces bordering on the square which undergoes Seiberg duality (see figure 17). In other words, since surface operators of the $4 \mathrm{D} \mathcal{N}=1$ theory compactified on $S_{(t)}^{1} \times M C_{q}$ correspond, in the small $S_{(t)}^{1}$ limit, to line operators of the $\mathcal{N}=2$ theory compactified on $\widetilde{S}_{(t)}^{1} \times M C_{q}$, we have determined the transformation rule (6.3) for the VEVs $\mathcal{V}_{f}$ in the small radius limit. 


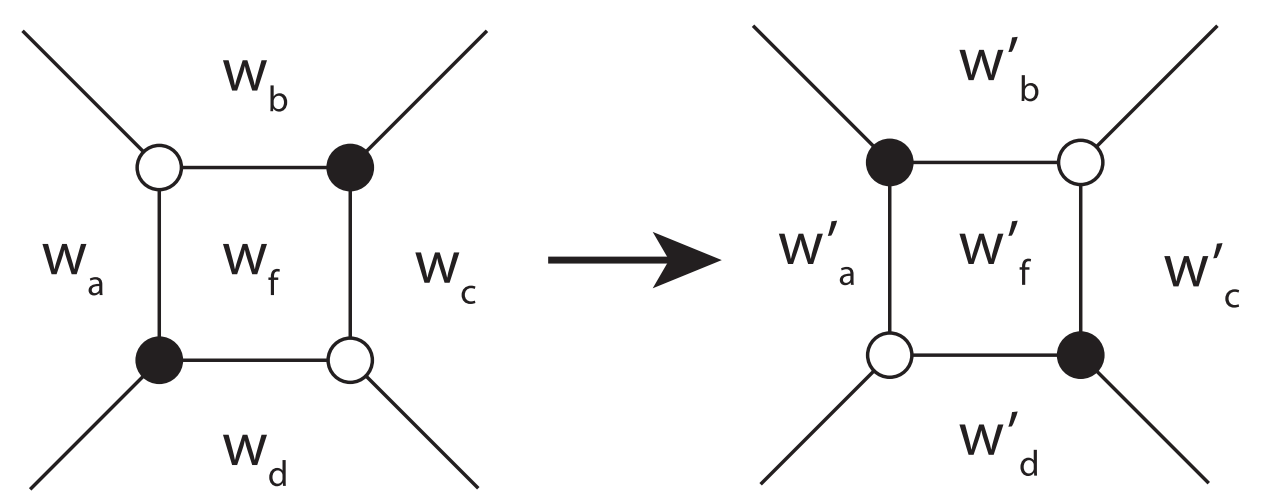

Figure 17: Depiction of the change of the VEVs of line operators in the dimensionally reduced theory under a Seiberg-like duality. The weights transform as $w_{f} \rightarrow w_{f}^{\prime}=w_{f}^{-1}, \quad w_{a, c} \rightarrow$ $w_{a, c}^{\prime}=w_{a, c}\left(1+w_{f}\right), \quad w_{b, d} \rightarrow w_{b, d}^{\prime}=w_{b, d}\left(1+w_{f}^{-1}\right)^{-1}$. This transformation is precisely what is required for face weights to specify a coordinate system for $\mathrm{Gr}_{k, n+k}^{\mathrm{tnn}}$.

Quite remarkably, in the combinatorics literature the transformation of the face weights in line (6.3) is precisely what is required in order for these variables to define coordinates of the totally non-negative Grassmannian [8] 10 In other words, we have shown how the VEVs of line operators associated with the reduction of surface operators in the 4D theory specify coordinates of the Grassmannian 11 Note that the mathematical literature on planar

\footnotetext{
${ }^{10}$ See [8] and Appendix A for details. The boundary measurement map is preserved under the square move if we simultaneously change the weights following (6.3).

${ }^{11}$ In the $\mathcal{N}=2$ context, this can be viewed as a statement about the "IR basis" of line operators discussed in 26]. Alternatively, one can work in terms of a "UV basis" of line operators associated with chords connecting punctures of the Gaiotto curve. In this alternative basis, these line operators specify Plücker coordinates of the Grassmannian, which transform as coordinates of the cluster algebra. See e.g. 27] for discussion of cluster algebras and coordinates of the Grassmannian in the math literature. It is helpful to illustrate this construction in the special case $y^{2}=x^{2}-a^{2}$, corresponding to a square dimer with four non-compact faces and one compact internal face. The compact face corresponds to the single node of the $A_{1}$ BPS quiver, i.e., it has a single $\mathcal{N}=2$ mass term. For our purposes, however, we can also include the additional $U(1)$ flavor symmetries coming from the $\mathcal{N}=1$ theory. Indeed, this is natural in the context of larger bipartite graph constructions where these additional faces are automatically compactified. In this simple setting, we can consider the related Gaiotto curve, which is given by a $\mathbb{P}^{1}$ with a fourth order pole at one marked point. This can be represented on the disk by marking four points on its boundary, and drawing chords between these marked points. The resulting six chords define contours on the Gaiotto curve, which are in turn mapped to the VEVs of line operators of the $\mathcal{N}=2$ theory. As found in [15], these coordinates form a cluster algebra, and satisfy the Plücker relation (see also [27]):
}

$$
x_{12} x_{34}+x_{14} x_{23}=x_{13} x_{24},
$$

where the points on the boundary of the disk are counter-clockwise ordered. From this identity, we see that there are only five independent line operators, which matches to the number of face 
networks [8] discusses only the case $q=1$. Our considerations suggest that there is an even richer $q$-deformation of these results.

\section{Conclusions}

The stringy realization of a gauge theory frequently provides powerful geometric insight into the strong coupling behavior of the field theory. In this paper we have seen that the recently proposed bipartite CFTs of [1] admit a stringy realization which explains the seemingly ad hoc rules found there. The geometry of the bipartite graph corresponds to one-dimensional flow lines which form the intersection locus in $\mathbb{C}^{2}$ between NS5-branes wrapping special Lagrangians and D5-branes wrapped on an algebraic curve. Varying the parameters of the background geometry with the asymptotics of the brane configuration held fixed corresponds to Seiberg duality in the field theory. In addition to providing a stringy explanation for the various non-trivial field theoretic dualities, we have also presented evidence that line operators of the 3D theory obtained from compactification on a circle provide coordinates for $\mathrm{Gr}_{k, n+k}^{\mathrm{tnn}}$. In the remainder of this section we discuss some further avenues of investigation.

In this work we have seen that the $\mathcal{N}=1$ bipartite theories defined by cells of $\mathrm{Gr}_{k, n+k}^{\mathrm{tnn}}$ exhibit a surprisingly rich phase structure as a function of $k$ and $n$. It is likely that our string construction, especially in the large $N$ limit may provide further insights into the IR behavior of these fixed points. Using our explicit brane construction, it would be quite instructive to construct and study a gravity dual description.

We have also provided a formal map between the geometry of related objects in an $\mathcal{N}=2$ theory and a corresponding $\mathcal{N}=1$ superconformal theory. In particular we have presented a conjectured relationship between the weights of faces, i.e. the coordinates of $\mathrm{Gr}_{k, n+k}^{\mathrm{tnn}}$ and VEVs of line operators in the 3D dimensionally reduced theory. It would be interesting to study this proposal further, even for the simple case of the $y^{2}=x^{n}$ theories.

In addition to their appearance in quiver gauge theories realized by D3-branes probing a toric Calabi-Yau, dimer models on a torus have also appeared in the study of topological strings on the same geometry [28, 29]. It is reasonable to ask whether topological string theory also makes use of planar dimers. Along these lines, it is natural to conjecture that the basic connection in this context involves the superconformal index of the $\mathcal{N}=1$ theories and the topological string partition function, as in [23,30]. Developing further the details of this correspondence would be illuminating on both fronts.

Finally, the appearance of bipartite graphs in diverse areas of theoretical physics is remarkable. In this work we have established a geometric connection between two such appearances in $\mathcal{N}=2$ and $\mathcal{N}=1$ theories. Similar bipartite graphs with a very different physical interpretation have also recently appeared in the study of $\mathcal{N}=4$ Super Yang-Mills

weights of the bipartite theory. We thank S. Cecotti for helpful discussions on this point. 
theory [31. It would be exciting to provide a physical link between these two appearances of planar bipartite graphs.

\section{Acknowledgments}

We thank the 10th Simons Summer Workshop in Mathematics and Physics, and the Simons Center for Geometry and Physics for hospitality and providing a stimulating environment where this work was initiated. We thank A. Neitzke for collaboration at an initial stage of this work, and for helpful discussions. We also thank N. Arkani-Hamed, J. Bourjaily, S. Cecotti, D. Morrison and B. Wecht for helpful discussions. The work of JJH is supported by NSF grant PHY-1067976, and that of CV is supported by NSF grant PHY-0244821. The work of DX is supported by DOE grant DE-FG02-90ER40542, and that of MY is supported by the Princeton Center for Theoretical Science.

\section{A Coordinates of $\mathbf{G r}_{k, n+k}^{\mathrm{tnn}}$}

In this Appendix we explain the mapping between coordinates of the totally non-negative Grassmannian $\mathrm{Gr}_{k, n+k}^{\mathrm{tnn}}$ and weights of the bipartite graph. The physical interpretation of the face weights is given in section [6. Our discussion follows that in [8], to which we refer the interested reader for further details.

We first define a perfect orientation. This is an orientation of edges of bipartite networks such that each black (white) vertex has one outgoing (incoming) arrow, with the rest incoming (outgoing), see figure 18 (a). For a given choice of the perfect orientation, we can consider the flow defined by the orientation of the arrows inside the corresponding network. Given an oriented network, $n$ of its legs are "sources" and point into the graph, while $k$ of these are sinks, i.e. attached to outgoing legs. We can verify that these numbers are the same $k, n$ defined in (2.1).

We can then define the "boundary measurement map":

$$
\text { Meas : } \operatorname{Net}_{k, n+k} \rightarrow \mathrm{Gr}_{k, n+k}^{\mathrm{tnn}}
$$

where Net $_{k, n+k}$ denotes the set of planar bipartite networks with given $k$ and $n$. To define this map, let us assign a positive weight to each edge of the network. Given a pair of a source and a sink (for a given choice of perfect orientation), we sum over all the possible oriented paths between them, whose weight is defined to be a (signed) product of all the edge weights as we go along the path. This defines a $k \times n$ matrix. By combining this with a $k \times k$ identity matrix, we can define a $k \times(n+k)$ matrix, which we define as the image of the boundary measurement map (A.1). 

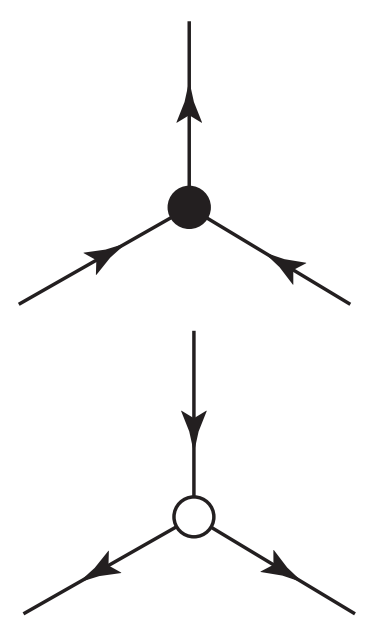

(a)

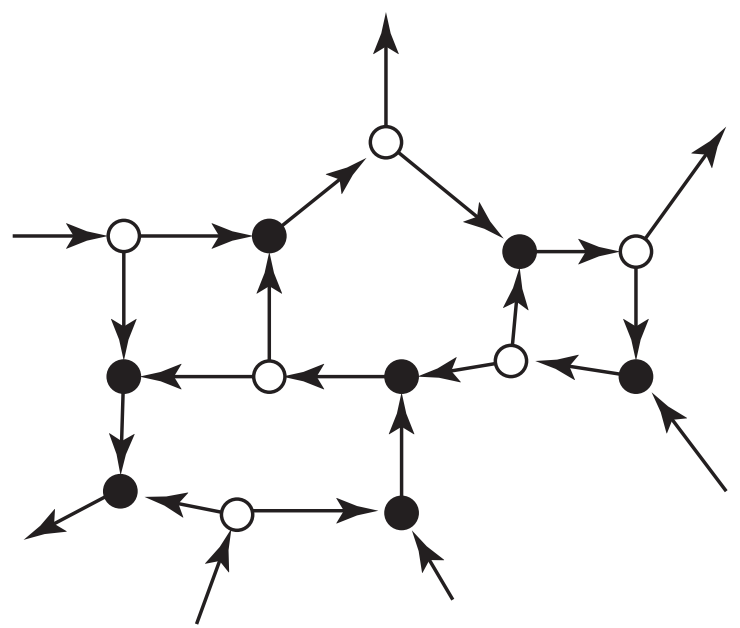

(b)

Figure 18: (a) A perfect orientation is a choice of orientation of a bipartite graph such that there are two outgoing (resp. incoming) arrows for a black (resp. white) vertex. (b) An example of a perfect orientation, with $k=3, n=4$.

The image of (A.1) fills out a particular cell specified by the network, and is independent of the choice of the perfect orientation chosen in the intermediate stage of the definition. Also, there is a redundancy in the edge assignments; All that really enters the matrix are products of weights associated with each face [8]. This is the face weight discussed in section 6 6. 12

\section{B Construction of Special Lagrangians}

In section 5 we presented a general construction of quiver gauge theories involving a D5-brane wrapping a holomorphic curve $\Sigma \subset \mathbb{C}^{2}$ defined by $y^{k}-x^{n}+$ (deformations $)=0$ and a class of NS5-branes wrapping special Lagrangian submanifolds. The basic assumption we made was that given a BPS flow line for the curve $\Sigma$, there exists a corresponding special Lagrangian in $\mathbb{C}^{2}$ which intersects $\Sigma$ along this flow line. In this Appendix we explain in more detail why these special Lagrangian submanifolds exist, and detail some of their properties. After this, we present an explicit construction in the case $y^{2}=x^{2}-a^{2}$.

\footnotetext{
12 In the language of cluster algebras, this is a change from cluster $x$-variables to cluster $y$ variables (coefficients).
} 


\section{B.1 Existence and Uniqueness}

In this subsection we show that given a BPS flow line on the curve $\Sigma$ defined by $y^{k}=$ $x^{n}+$ (deformations), there is a corresponding special Lagrangian $L$ which intersects $\Sigma$ along this flow line.

The basic observation is that $\mathbb{R}^{4}$ is hyperkahler, admitting an entire sphere's worth of complex structures. We can specify a sLag $L$ as an analytic subvariety with respect to another complex structure, and therefore give a characterization of possible intersections between $L$ and the original curve $\Sigma$ in terms of holomorphic geometry of the rotated complex structure. We begin by introducing the real coordinates $x_{i}$ and $y_{i}$ :

$$
x=x_{1}+i x_{2} \text { and } y=y_{1}+i y_{2} .
$$

In this complex structure, the holomorphic two-form is $\Omega=d x \wedge d y$ and the Kähler form is $J=\frac{i}{2}(d x \wedge d \bar{x}+d y \wedge d \bar{y})$. This is only one of many possible choices of complex structure. We could alternatively take $\operatorname{Re} e^{-i \theta} \Omega=\widetilde{J}=\frac{i}{2}(d u \wedge d \bar{u}+d v \wedge d \bar{v})$ to be the Kähler form in a rotated complex structure with holomorphic coordinates $u$ and $v$ :

$$
\begin{aligned}
& u=\operatorname{Re} x+i \operatorname{Re} e^{-i \theta} y=x_{1}+i\left(\cos \theta y_{1}+\sin \theta y_{2}\right) \\
& v=\operatorname{Im} x-i \operatorname{Im} e^{-i \theta} y=x_{2}+i\left(\sin \theta y_{1}-\cos \theta y_{2}\right)
\end{aligned}
$$

Our candidate sLags are therefore given by the zero set of an analytic function $g(u, v)$ :

$$
L=\{g(u, v)=0\}
$$

in the rotated complex structure.

This coordinate system is quite helpful in describing the special Lagrangians which intersect $y^{k}=x^{n}$ along BPS flow lines. Based on the BPS flow equation $y d x=\alpha d t$, we see that since the $t$ coordinate is a real parameter, we must demand:

$$
\operatorname{Im}\left[e^{i \theta} x^{(n+k) / k}\right]=0 .
$$

In other words, on one of the sheets of the cover for $\Sigma$, we have:

$$
\begin{aligned}
& x=e^{-i k \theta /(n+k)} \xi_{n+k} r_{x}, \\
& y=e^{-i n \theta /(n+k)} \xi_{n+k}^{-1} r_{y} .
\end{aligned}
$$

For $r_{x}$ and $r_{y}$ real with $\xi_{n+k}$ an $(n+k)^{\text {th }}$ root of unity. The other sheets are obtained by multiplying $y$ by a $k^{\text {th }}$ root of unity.

Without loss of generality, we can set $\theta=0$, and work with respect to the same sheet 
used above. In terms of the coordinates $u$ and $v$, we have:

$$
\begin{aligned}
& u=\operatorname{Re} x+i \operatorname{Re} y=x_{1}+i y_{1}, \\
& v=\operatorname{Im} x-i \operatorname{Im} y=x_{2}-i y_{2} .
\end{aligned}
$$

Writing $\xi_{n+k}=\cos \vartheta+i \sin \vartheta$, we therefore have:

$$
\begin{aligned}
& u=\cos \vartheta r_{x}+i \cos \vartheta r_{y}, \\
& v=\sin \vartheta r_{x}+i \sin \vartheta r_{y} .
\end{aligned}
$$

In other words the sLag is specified by:

$$
\frac{u}{v}=\cot \vartheta
$$

The corresponding algebraic equation describing the sLag is:

$$
g(u, v)=\prod_{j=1}^{n+k}\left(u \sin \frac{2 \pi j}{n+k}-v \cos \frac{2 \pi j}{n+k}\right) .
$$

Our discussion so far has involved the explicit form of the sLag in the limit where all lower order deformations of $\Sigma$ have been switched off. Once we switch on deformations of $\Sigma$, the explicit parameterization of the sLag will be quite complicated. Nevertheless, it is still possible to argue for the existence of a sLag given a particular BPS flow line. We work away from points of ramification of $\Sigma$. In this case, the BPS flows are characterized by a one-dimensional path on $\Sigma$.

To construct the profile of the sLag in a local patch surrounding the BPS flow line, we proceed as follows. Consider first the trajectory of the curve in $\mathbb{R}^{4}$ viewed as $\mathbb{C}^{2}$ with respect to the rotated complex structure $u(t), v(t)$ :

$$
\begin{aligned}
\gamma: \mathbb{R} \rightarrow \mathbb{R}^{4} \\
t \mapsto\left(u_{1}(t), u_{2}(t), v_{1}(t), v_{2}(t)\right) .
\end{aligned}
$$

To extend this curve to a special Lagrangian, we locally "fatten" this one-dimensional space to a two-dimensional manifold. In other words, having specified a trajectory, we need to pick one direction normal to the curve such that the resulting sLag is locally fixed. The tangent vector along the curve is given by the $\mathbb{R}^{4}$ vector $\left(u_{1}^{\prime}(t), u_{2}^{\prime}(t), v_{1}^{\prime}(t), v_{2}^{\prime}(t)\right)$. The normal directions sweep out an $\mathbb{R}^{3}$ within this space. Out of these three directions, the one corresponding to the normal direction is the one compatible with the complex structure specified by $u$ and $v$. This choice is unique, and is obtained by acting via $\widetilde{J}$, the complex 
structure in the rotated basis. More precisely, there is a group of $U(2)$ transformations on the coordinates $\left(u^{\prime}(t), v^{\prime}(t)\right)$ which leave the complex structure unchanged. Of this group of $U(2)$ transformations, only a $U(1)$ subgroup also leaves the holomorphic two-form invariant. The holomorphic two-form and Kähler form in the rotated complex structure are invariant under the rephasing:

$$
\left(u^{\prime}, v^{\prime}\right) \rightarrow\left(e^{i \phi} u^{\prime}, e^{-i \phi} v^{\prime}\right) .
$$

Hence, this circle action locally produces a two-manifold which is special Lagrangian. Thus, in addition to the time coordinate $t$, we can also introduce a fixed normal direction $n$. The coordinates $u$ and $v$ are now functions of two coordinates $t$ and $n$. Let us note in passing that this local of construction of special Lagrangians works in higher dimensions as well 13

In other words, once we find a special Lagrangian passing through the prescribed 1cycle, there is no remaining choice; it is the unique option available. Locally, we have also seen that the existence of a 1-cycle does not lead to any obstruction to the construction of the corresponding special Lagrangian, so we conclude that at least locally, our special Lagrangians exists. Finally, even though near the curve $\Sigma$ the components of the sLag are smooth, it could indeed happen that far from the holomorphic curve, the behavior of the sLag looks singular. This is not really an issue for our present purposes since we are only interested in the local behavior of the sLag near $\Sigma$.

\section{B.2 Explicit Example}

Having given a general local construction of such sLags, it is helpful to consider an explicit example. We consider $\Sigma$ given by:

$$
y^{2}=x^{2}-a^{2}
$$

for $a>0$. In this section we construct the sLags which intersect $\Sigma$ along the BPS flow lines of $\Sigma$, which are specified by the constant phase trajectories of $y d x=\alpha d t$ for $\alpha$ a complex phase. Throughout, we restrict attention to the case $\alpha=1$, so that the phases of $a$ and $\alpha$ are aligned.

A helpful physical model to keep in mind is complexified quantum mechanics with momentum $y$ and position $x$ (see footnote 8). Studying the BPS flow lines amounts to working in the semi-classical approximation for this system. To integrate $y d x$, it is helpful to work

\footnotetext{
${ }^{13}$ See e.g. [32, 33] and [34] for a review. Essentially quoting from [34, given a real analytic $(m-1)$ - dimensional submanifold $P$ of $\mathbb{C}^{m}$ with a non-vanishing section of $\Lambda^{m-1} T P$ which we denote by $\chi$, we can construct a one-parameter family of maps $\phi_{t}: P \rightarrow \mathbb{C}^{m}$, and a corresponding special Lagrangian satisfying the ODE:

$\left(\frac{d \phi_{t}}{d t}\right)^{b}=\left(\phi_{t}\right)_{*}(\chi)^{a_{1} \ldots a_{m-1}}(\operatorname{Re} \Omega)_{a_{1} \ldots a_{m}} g^{a_{m} b}$. Locally, this yields a special Lagrangian parameterized as $L=\left\{\phi_{t}(p)\right.$ for $t \in \mathbb{R}$ and $\left.p \in P\right\}$.
} 
in terms of the coordinates $X$ and $Y$ specified as:

$$
\begin{aligned}
& x=a \cosh X, \\
& y=a \sinh Y .
\end{aligned}
$$

This is a non-singular coordinate transformation near the origin. At large coordinate values, it develops some singularities. However, we view the complexified phase space as a region in $\mathbb{C}^{2}$, i.e., we delete the essential singularities in $X$ and $Y$ space.

In terms of these coordinates, the holomorphic curve reads as:

$$
\sinh ^{2} Y=\sinh ^{2} X
$$

In other words, we have:

$$
Y= \pm X
$$

for the two sheets of the Riemann surface.

Next, we note that in this coordinate system, the WKB phase also simplifies:

$$
\int y d x=\frac{a^{2}}{4}(\sinh 2 X-2 X)
$$

Since we are assuming $a>0$ writing out $X=X_{1}+i X_{2}$, the condition that $\int y d x$ is real can be written as:

$$
\cosh \left(2 X_{1}\right) \sin \left(2 X_{2}\right)=2 X_{2} .
$$

This yields two branches. Either we have $X_{2}=0$ or, if $X_{2}$ does not vanish, we obtain a more complicated trajectory. We are interested in the branch which intersects $X_{2}=0$, as this is where a consistent flow can terminate. We can also identify the location of the other flow lines. This corresponds to taking $\sin X_{2} \simeq 1$ and $X_{1} \rightarrow \infty$. In this limit, we approach an asymptotic trajectory where $\operatorname{Re} x \rightarrow 0$ and $\operatorname{Im} x \rightarrow \infty$.

This can be viewed as the analytic continuation of the inverted harmonic oscillator in the complex $x$-plane. A particle moving along this path is following a steepest descent along which the phase of the path integral can remain constant. Having solved for the trajectory in the $X$ coordinates, it follows that we can also deduce the flow in the $Y$ coordinates, via equation (B.21). The appropriate trajectory on the top sheet of the Riemann surface is therefore satisfied by the equations:

$$
\cosh \left(2 X_{1}\right) \sin \left(2 X_{2}\right)=2 X_{2}, Y_{1}=X_{1} \text {, and } Y_{2}=X_{2} \text {. }
$$

Let us now turn to a parametrization of the sLag in terms of our $u$ and $v$ coordinates. Our aim will be to find an analytic expression in the $u$ and $v$ coordinates which intersects 
the holomorphic curve along the specified BPS flow lines. Our strategy for doing this will be to consider an explicit presentation for $u$ and $v$ as functions of the coordinates $X_{1}$ and $X_{2}$. Using our relation between $X_{1}$ and $X_{2}$, we will aim to convert this into an analytic relation between $u$ and $v$. To do this, we view $u\left(X_{1}, X_{2}\right)$ and $v\left(X_{1}, X_{2}\right)$ as functions of two complex variables $X_{1}$ and $X_{2}$, i.e., we analytically continue the relation of equation (B.24) to $\mathbb{C}^{2}$.

To this end, let us begin by first writing out in terms of $a, X_{1}$ and $X_{2}$, the form of the coordinates $u$ and $v$ in the rotated complex structure:

$$
\begin{aligned}
& u=\operatorname{Re} x+i \operatorname{Re} y=a\left(\cos X_{2} \cosh X_{1}+i \cos X_{2} \sinh X_{1}\right) \\
& v=\operatorname{Im} x-i \operatorname{Im} y=a\left(\sin X_{2} \sinh X_{1}-i \sin X_{2} \cosh X_{1}\right)
\end{aligned}
$$

It is helpful to consider the analytic expressions:

$$
\frac{i v}{u}=\tan X_{2}, \quad u^{2}-v^{2}=a^{2}\left(1+i \sinh 2 X_{1}\right)
$$

Eliminating the appearance of $X_{1}$ and $X_{2}$, we obtain the equation for the sLag:

$$
g_{\gamma}(u, v)=0
$$

where

$$
g_{\gamma}(u, v) \equiv v-u \tanh \left(\frac{u v}{a^{2}} \sqrt{\frac{v^{2}-u^{2}+2 a^{2}}{u^{2}-v^{2}}}\right) .
$$

As we originally anticipated, the deformation involves a transcendental function. This means that there will actually be infinitely many branches of this function, and so the sLag will actually intersect the holomorphic curve $\Sigma$ at infinitely many lines. In other words, in addition to the original BPS flow lines, we have an infinite set of additional intersections between our holomorphic curve and our sLag.

Physically, however, this further subdivision of the $x$-plane is meaningless. Indeed, these regions are identified with a single gauge group in the $4 \mathrm{D}$ field theory. In order to have treated these regions as defining distinct gauge group factors, we would need to have a field theory mode, i.e. a light string in the theory which could condense to lift this region away from the rest. For the regions of the original bipartite graph, we can see that such modes exist and are the bifundamentals between the flavor branes. However, we also see that there is no consistent way to assign a collection of bifundamentals to the other regions. Indeed, if we had tried to assign a different gauge group factor to each region we would have obtained an anomalous gauge group factor. 


\section{References}

[1] D. Xie and M. Yamazaki, "Network and Seiberg Duality," arXiv:1207.0811 [hep-th].

[2] A. Hanany and K. D. Kennaway, "Dimer models and toric diagrams," arXiv:hep-th/0503149 [hep-th].

[3] S. Franco, A. Hanany, K. D. Kennaway, D. Vegh, and B. Wecht, "Brane dimers and quiver gauge theories," JHEP 0601 (2006) 096, arXiv: hep-th/0504110 [hep-th].

[4] S. Franco, A. Hanany, D. Martelli, J. Sparks, D. Vegh, et al., "Gauge theories from toric geometry and brane tilings," JHEP 0601 (2006) 128, arXiv:hep-th/0505211 [hep-th].

[5] B. Feng, Y.-H. He, K. D. Kennaway, and C. Vafa, "Dimer Models from Mirror Symmetry and Quivering Amoebae," Adv. Theor. Math. Phys. 12 (2008) 489-545, arXiv:hep-th/0511287 [hep-th].

[6] K. D. Kennaway, "Brane Tilings," Int.J.Mod.Phys. A22 (2007) 2977-3038, arXiv:0706.1660 [hep-th].

[7] M. Yamazaki, "Brane Tilings and Their Applications," Fortsch.Phys. 56 (2008) 555-686, arXiv:0803.4474 [hep-th].

[8] A. Postnikov, "Total Positivity, Grassmannians, and Networks," arXiv:0609764 [math].

[9] S. Franco, "Bipartite Field Theories: from D-Brane Probes to Scattering Amplitudes," arXiv:1207.0807 [hep-th].

[10] H. Ooguri and M. Yamazaki, "Crystal Melting and Toric Calabi-Yau Manifolds," Commun.Math.Phys. 292 (2009) 179-199, arXiv:0811.2801 [hep-th].

[11] K. A. Intriligator and B. Wecht, "The Exact superconformal R symmetry maximizes a," Nucl. Phys. B667 (2003) 183-200, arXiv:hep-th/0304128 [hep-th].

[12] D. Kutasov and A. Schwimmer, "On duality in supersymmetric Yang-Mills theory," Phys.Lett. B354 (1995) 315-321, arXiv:hep-th/9505004 [hep-th].

[13] D. Kutasov, A. Parnachev, and D. A. Sahakyan, "Central charges and U(1)(R) symmetries in N=1 superYang-Mills," JHEP 0311 (2003) 013, arXiv:hep-th/0308071 [hep-th]. 
[14] K. A. Intriligator and B. Wecht, "RG fixed points and flows in SQCD with adjoints," Nucl.Phys. B677 (2004) 223-272, arXiv:hep-th/0309201 [hep-th].

[15] S. Cecotti, A. Neitzke, and C. Vafa, "R-Twisting and 4d/2d Correspondences," arXiv:1006.3435 [hep-th].

[16] M. Alim, S. Cecotti, C. Cordova, S. Espahbodi, A. Rastogi, et al., "BPS Quivers and Spectra of Complete N=2 Quantum Field Theories," arXiv:1109.4941 [hep-th].

[17] D. Xie, "Network, Cluster coordinates and N=2 theory I," arXiv:1203.4573 [hep-th].

[18] D. Gaiotto, G. W. Moore, and A. Neitzke, "Spectral networks," arXiv:1204.4824 [hep-th].

[19] D. Gaiotto, G. W. Moore, and A. Neitzke, "Spectral Networks and Snakes," arXiv:1209.0866 [hep-th].

[20] A. D. Shapere and C. Vafa, "BPS structure of Argyres-Douglas superconformal theories," arXiv:hep-th/9910182 [hep-th].

[21] H. Ooguri and C. Vafa, "Summing up D-Instantons," Phys. Rev. Lett. 77 (1996) 3296-3298, arXiv:hep-th/9608079 [hep-th].

[22] E. Witten, "A New Look At The Path Integral Of Quantum Mechanics," arXiv:1009.6032 [hep-th].

[23] M. Yamazaki, "Quivers, YBE and 3-manifolds," JHEP 1205 (2012) 147, arXiv:1203.5784 [hep-th].

[24] Y. Terashima and M. Yamazaki, "Emergent 3-manifolds from 4d Superconformal Indices," Phys.Rev.Lett. 109 (2012) 091602, arXiv:1203.5792 [hep-th].

[25] S. Cecotti, C. Cordova, and C. Vafa, "Braids, Walls, and Mirrors," arXiv:1110.2115 [hep-th].

[26] D. Gaiotto, G. W. Moore, and A. Neitzke, "Framed BPS States," arXiv:1006.0146 [hep-th].

[27] J. S. Scott, "Grassmannians and cluster algebras," Proc. London. Math. Soc. (3) 92 (2006) 345-380, math/0311148.

[28] A. Okounkov, N. Reshetikhin, and C. Vafa, "Quantum Calabi-Yau and classical crystals," Progr.Math. 244 (2006) 597, arXiv:hep-th/0309208 [hep-th]. 
[29] H. Ooguri and M. Yamazaki, "Emergent Calabi-Yau Geometry," Phys.Rev.Lett. 102 (2009) 161601, arXiv:0902.3996 [hep-th].

[30] C. Vafa, "Supersymmetric Partition Functions and a String Theory in 4 Dimensions," arXiv:1209.2425 [hep-th].

[31] N. Arkani-Hamed, J. L. Bourjaily, F. Cachazo, A. B. Goncharov, A. Postnikov, et al., "Scattering Amplitudes and the Positive Grassmannian," arXiv:1212.5605 [hep-th].

[32] D. Joyce, "Constructing special Lagrangians $m$-folds in $\mathbb{C}^{m}$ by evolving quadrics," Math. Ann. 320 (2001) 757-797, arXiv:math.DG/0008155 [math.DG].

[33] D. Joyce, "Evolution equations for special Lagrangian 3-folds in $\mathbb{C}^{3}$," Global Anal. Geom. 20 (2001) 345-403, arXiv:math.DG/0010036 [math.DG].

[34] D. Joyce, "Lectures on Calabi-Yau and special Lagrangian geometry," arXiv:math/0108088 [math-dg]. 